\title{
1 Cognitive and cultural models in psychological science: A 2 tutorial on modeling free-list data as a dependent variable in
}

\author{
Theiss Bendixen ${ }^{\mathrm{a}} \&$ Benjamin Grant Purzycki ${ }^{\mathrm{a}}$ \\ a Department of the Study of Religion, Aarhus University, Denmark
}

\begin{abstract}
Assessing relationships between culture and cognition is central to psychological science. To this end, free-listing is a useful methodological instrument. To facilitate its wider use, we here present the free-list method along with some of its many applications and offer a tutorial on how to prepare and statistically model free-list data as a dependent variable in Bayesian regression using openly available data and code. We further demonstrate the real-world utility of the outlined workflow by modeling within-subject agreement between a free-list task and a corollary item response scale on religious beliefs with a cross-culturally diverse sample. Overall, we fail to find a reliable statistical association between these two instruments, an original empirical finding that calls for further inquiry into identifying the cognitive processes that item response scales and free-list tasks tap into. Throughout, we argue that free-listing is an unambiguous measure of cognitive and cultural information and that the free-list method therefore has broad potential across the social sciences aiming to measure and model individual-level and cross-cultural variation in mental representations.
\end{abstract}

Keywords: cognitive anthropology, cross-cultural psychology, free-listing, item response scale, zero-one inflated beta regression 


\section{Introduction}

Assessing relationships between culture and cognition is central to psychological science. This is further emphasized by the recent surge in cross-cultural behavioral and psychological research (e.g., Barrett, 2020; Broesch et al., 2020). Developing and applying valid measures of inter-individual and inter-group variation in cognitive and cultural models -including values, attitudes, and beliefs - is therefore critical (D'Andrade, 1987, 1995).

To that end, item response scales are ubiquitous across the social sciences. However, item response scales are not unproblematic ${ }^{1}$. For instance, psychometricians have long noted that arbitrary structural features of scales such as the number, order, and difficulty of response options and items systematically influence participants' response patterns (e.g., Haaf, Merkle, \& Rouder, 2020; Krosnick \& Presser, 2009; Panter, Tanaka, \& Wellens, 1992; Şahin, 2021). In cross-cultural settings, item response scales have also proven difficult to administer in non-numerate field sites (Purzycki et al., 2016a; Purzycki et al., 2022; Quinlan, 2017). Further, while cognitive anthropologists have continually emphasized the importance of obtaining cross-task and construct validity using a diversity of instruments (e.g., D’Andrade, 1995; Dressler, Borges, Balieiro, \& Santos, 2005; Furlow, 2003; Romney, Weller, \& Batchelder, 1986), item scales are rarely validated using alternative methods of measurement.

One such alternative method is free-listing. In a free-list task, participants are simply invited to list their associations on some topic. The list can-but need not-be capped at some limit. The listed items are then thematically coded according to a specified level of granularity adapted to the particular research goal at hand. Items listed earlier are typically easier to access or recall, and thus constitute a form of cognitive salience (Chaves et al., 2019; Romney \& d'Andrade, 1964; Thompson \& Juan, 2006). Further, given a valid topic, items higher up on an individual's list tend to also be more prevalent across lists drawn from the same sample, indicating the free-list task as an unambiguous measure of cultural information (e.g., Bendixen, Apicella, et al., forthcoming; Purzycki \& Bendixen, 2020; Purzycki, Pisor, et al., 2018; Quinlan, 2017; see D'Andrade, 1995, pp. 212-217 for a general discussion of "cultural consensus"). These properties make free-listing a promising measure of individual and cross-cultural cognitive models in psychological science.

While free-list data lend themselves to a host of analytic approaches (e.g., Purzycki, forthcoming; Quinlan, 2017; Smith, 1993), free-lists are often mainly used for descriptive or exploratory purposes or for informing downstream item scale construction. Free-listing has seen widespread use in a range of sub-disciplines within cognitive anthropology and psychology (for one overview, see Bernard, 2018, pp. 235-238), including - but not limited to - ethnobiology (e.g., Chaves et al., 2019; Fremout et al., 2021; McNamara \& Wertz, 2021; Quinlan, 2005), linguistics (e.g., Henley, 1969; Smith \& Borgatti, 1997; Smith, Furbee, Maynard, Quick, \& Ross, 1995), as well as the study of spiritual and theistic beliefs and practices (e.g., Atran et al., 2002; McNamara, Senanayake, Willard, \& Henrich, 2021; Purzycki, 2011, 2013; Purzycki \& Holland, 2019; Purzycki \& Kulundary, 2018; Singh \& Henrich, 2020; Singh, Kaptchuk, \& Henrich, 2021; Turpin \& Stanford, 2020; Willard, Baimel,

\footnotetext{
${ }^{1}$ For a principled approach to measuring cultural models using item response theory, see Bunce and McElreath (2017).
} 
Turpin, Jong, \& Whitehouse, 2020). But recent work has demonstrated the promise of more principled statistical modeling of free-list data, for instance as an independent variable in regression models (e.g., Kundtová Klocová, Lang, Maňo, Kundt, \& Xygalatas, 2022; Purzycki, Pisor, et al., 2018; White \& Norenzayan, 2021) and in semantic (e.g., Levine, Muthukrishna, Chan, \& Satterfield, 2015) and social (e.g., Redhead, McElreath, \& Ross, 2021) network analysis. Some studies employ free-list data as a dependent variable and, for instance, compare the number of items listed between groups (e.g., Brewer, Garrett, \& Rinaldi, 2002) or model the presence of some target item (e.g., Purzycki \& Bendixen, 2020; Purzycki, Stagnaro, \& Sasaki, 2020; White \& Norenzayan, 2021) as a function of some predictor(s) or following experimental manipulation.

When research questions pertain to the psychological salience of some particular concept or item, coded free-list data can be converted into salience scores (Quinlan, 2017; Smith, 1993; Smith \& Borgatti, 1997). Salience scores range from 0 (i.e., target item not listed) to 1 (i.e., target item listed first) with intermediate values representing the target item's order of listing (i.e., a higher score means that the target item is listed earlier), taking into account the total number of items that a participant lists (see Part One for formula). However, distributions of data bound by 0 and 1 , and where 0 and 1 are valid values, are generally not compatible with common assumptions of popular statistical procedures (Cribari-Neto \& Santos, 2019; Douma \& Weedon, 2019; Kubinec, 2022; F. Liu \& Eugenio, 2018; F. Liu \& Kong, 2015; P. Liu, Yuen, Wu, Tian, \& Li, 2020; Menezes, Mazucheli, \& Bourguignon, 2021; Ospina \& Ferrari, 2012; Queiroz \& Lemonte, 2021; Santos \& Bolfarine, 2015; Swearingen, Castro, \& Bursac, 2012). This poses a challenge to analysts who want to model free-list salience scores as a dependent variable and calls for general guidelines on how to go about modeling salience scores and similar zero-one inflated and bounded data. We offer one such set of guidelines here.

By way of illustrating the usefulness of the free-list task and salience calculations, consider a recent study by Purzycki, Pisor, et al. (2018). To assess cross-cultural mental representations of moral norms, the research group administered a free-list task across eight ethnographically diverse field sites. Participants were asked to freely list the kinds of things that makes a "good" and "bad" person, respectively, and responses were subsequently cleaned, coded and salience scored. This workflow allowed the researchers to achieve a number of objectives not easily achieved with pre-fabricated instruments, such as item response scales: First, site-specific salience of particular moral norms could be explored emically, that is from the perspective of the local participants, without any assumptions a priori about the content and structure of local moral norms. Second, cross-culturally recurrent responses such as generosity and honesty (i.e., "moral universals") were uncovered and then compared to - and found to be only partially overlapping with - theoretical approaches to human morality, such as Moral Foundations Theory (Graham et al., 2013) and Morality-as-Cooperation (Curry, Whitehouse, \& Mullins, 2019), derived from more structured, item-based research designs. Thirdly, individual-level free-list data were linked up with prosocial decision-making in an economic game in order to infer a statistical relationship between explicit moral norms and corresponding moral behavior. This study, then, highlights how the free-list task, together with formalized post-processing such as salience scoring and principled modeling, can facilitate a bridging of qualitative and quantitative research designs and in so doing 
open up novel avenues for social scientific inquiry into human behavior and cognition.

This paper has two main parts. In Part One, we outline how to calculate and statistically model salience scores from a free-list task as a dependent variable in Bayesian regression (for general introductions to Bayesian data analysis, see Gelman, Carlin, Stern, \& Rubin, 2013; A. A. Johnson, Ott, \& Dogucu, 2022; Kruschke, 2014; Kruschke \& Liddell, 2018; McElreath, 2020) ${ }^{2}$. Specifically, we suggest the zero-one inflated beta distribution as likelihood model and illustrate its utility compared to popular alternatives using simulated synthetic data. Throughout, we primarily focus on how to model salience scores as they are a commonly used transformation that conveniently integrates relevant information (i.e., item list position and total list length) into a single metric. However, we also discuss approaches to modeling free-list data without transforming the data into salience scores and provide practical introductions to these alternatives in the supplementary materials.

In Part Two, we apply the presented tools in a real-world case study, including practical steps in how to prepare coded free-list data for modeling. The motivating case study pertains to individual-level data on the moral concerns ascribed to locally relevant deities, a central topic in the cognitive and evolutionary study of religion (for a recent review, see Bendixen, Lightner, \& Purzycki, forthcoming). Specifically, we model within-subject agreement between a free-list task and a corollary item response scale in a cross-culturally diverse sample and overall, we fail to find a reliable statistical association between these two instruments. This original empirical finding calls for further inquiry into identifying the cognitive processes that item response scales and free-list tasks reflect. We conclude with a call for psychological researchers to consider the free-list task as an alternative or complementary instrument to item response scales for measuring cognitive and cultural models of mental representations.

To render the tutorial maximally useful, all code is written in the popular open-source programming language $\mathrm{R}$ ( $\mathrm{R}$ Core Team, 2021, version 4.1.2), and we integrate key code chunks into the main text throughout. Modeling is implemented using the brms package (Bürkner, 2017, 2018, 2021), an interface to the probabilistic programming language Stan (Carpenter et al., 2017; Stan Development Team, 2021), and free-list data preparation is handled via AnthroTools (Jamieson-Lane \& Purzycki, 2016; Purzycki \& Jamieson-Lane, 2016). Data wrangling and plotting are primarily facilitated by the tidyverse (Wickham et al., 2019), ggplot2 (Wickham, 2016), tidybayes (Kay, 2022), and bayesplot (Gabry \& Mahr, 2021; Gabry, Simpson, Vehtari, Betancourt, \& Gelman, 2019) packages ${ }^{3}$. Data and code to reproduce the full manuscript and supplementary sections, written in $\mathrm{R}$ Markdown (Allaire et al., 2021; Xie, Allaire, \& Grolemund, 2018), are publicly available ${ }^{4}$.

\footnotetext{
${ }^{2}$ See also Kurz' (Kurz, 2021a, 2021b) code translations of Kruschke (2014) and McElreath (2020).

${ }^{3}$ See Supplementary Section D for a list of R packages, their dependencies, and version number used for this project, created using grateful (Rodríguez-Sánchez, Jackson, \& Hutchins, 2022).

${ }^{4}$ The package renv (Ushey, 2022) ensures a reproducible package environment.
} 


\section{Part One: Simulating and modeling zero-one inflated and bounded data}

\subsection{Salience calculations}

A listed item's salience score depends on both its listed order and the total number of listed items per participant. To calculate a salience score, an item's order number, $k$, is subtracted from 1 plus the total number of items a participant listed, $n$. This number is then divided by the total number of items listed, $\frac{n+1-k}{n}$. All items listed first thus get an item salience of 1. Items listed earlier have a higher salience score (assuming equal list lengths) and are typically easier to access or recall (e.g., Chaves et al., 2019; Quinlan, 2017; Smith, 1993; Smith \& Borgatti, 1997). For salience calculations, we use the AnthroTools package (Jamieson-Lane \& Purzycki, 2016; Purzycki \& Jamieson-Lane, 2016) with the MAX_SALIENCE table type function, which takes the maximum salience of the listed items in cases, where subjects listed the same item more than once. This is particularly useful when participants can list multiple items that would subsequently be coded similarly (e.g., in the context of our case study, "theft", "murder", and "deceit" might all be coded as "Morality"; see Part Two and Bendixen, Apicella, et al. (forthcoming) for details). These individual-level salience scores can then be modeled as the dependent variable as a function of some predictor(s). We illustrate a practical implementation in $\mathrm{R}$ in Part Two.

\subsection{The zero-one inflated beta distribution}

Since salience scores range continuously from 0 to 1 , and since 0 (item not listed) and 1 (item listed first) are valid values, we suggest modeling the outcome with a zero-one inflated beta (ZOIB) likelihood distribution with conventional link functions (for formal details, see F. Liu \& Eugenio, 2018; F. Liu \& Kong, 2015; Ospina \& Ferrari, 2012). The ZOIB is a mixture model determined by four parameters: the mean and the precision of the beta distribution; a "zero-one inflation" parameter (i.e. the probability that an observation is either 0 or 1 ); and a "conditional one inflation" parameter (i.e. the probability that, given an observation is 0 or 1 , the observation is 1 ). This specification captures the entire range of possible values while still being constrained between zero and one.

Other common approaches to modeling 0 to 1 bounded outcome data include a Gaussian or a regular beta distribution. However, among other limitations, the former depends on the assumption of normality, an assumption that rarely holds in real-world data of this kind and is therefore unlikely to provide a good fit to data in cases where the density of the distribution is not symmetrically centered around a midpoint. Further, if the Gaussian distribution is not truncated, the model can predict values outside the valid range of $0-1$. On the other hand, while the beta distribution is more flexible than the normal distribution in that it can capture skewed and non-Gaussian densities, 0 and 1 are not valid values, and hence it is an inappropriate likelihood function for zero and/or 1 inflated data. One proposed solution to this problem is the "transformed beta" distribution, where 0s and 1s are transformed to be, respectively, just above 0 (e.g., 0.001) and below 1 (e.g., 0.999). However, transforming data is arguably unprincipled and fails to capture a key feature of the data-generating process, particularly if the probability of zeros and/or ones are of theoretical interest. In the next section, we informally compare the fit of ZOIB to a Gaussian and transformed beta model using simulated zero-one inflated and bounded data. 
An alternative likelihood model for this kind of data is the recently proposed "ordered beta" (Kubinec, 2022). Compared to the ZOIB, which splits the "effect" estimates into several parameters, the ordered beta yields a single "effect" estimate in that it uses the same predictive model for both discrete and continuous outcomes. However, if the probability of zeros and/or ones has a natural interpretation (as is the case for free-list salience scores, where 1 reflects "item listed first" and 0 equals "item not listed") and is of theoretical interest, the ZOIB might still be preferable. In Supplementary Section A, we demonstrate an implementation of the ordered beta and, in the context of the case study of the present paper at least, the ZOIB and the ordered beta yield similar results and posterior predictions.

\subsection{Synthetic data and analysis}

To illustrate the utility of modeling salience scores (and similar data) using the ZOIB, we simulate and analyze zero-one inflated and bounded data, briefly discuss the interpretation and some simple post-processing of the output from brms, and compare the model to alternative likelihood models, namely the Gaussian distribution and the transformed beta. We finally discuss some limitations to the ZOIB and briefly consider some alternative modeling options that do not rely on transforming free-list data into salience scores.

1.3.1 Simulation. We simulate zero-one inflated and bounded data from a ZOIB for two groups, and model the "effect" of group on the distribution of the outcome variable. Imagine, for instance, that these are salience scores from a free-list task administered at two field sites. We are then interested in whether a target item is listed earlier (i.e., is more salient) at one field site compared to the other. The data is stored in sim_dat. Assuming default priors (see Part Two for some explication on prior choice), this simple model is implemented in brms as follows ${ }^{5}$.

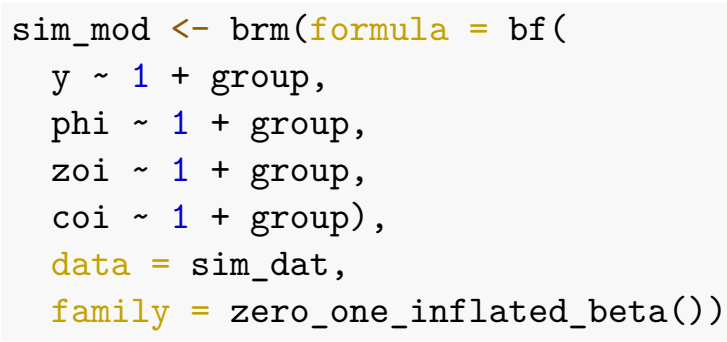

Recall that we are predicting the outcome with four parameters. Here, y represents the mean of the beta distribution. In brms, unlike the remaining parameters which have set names, the mean parameter does not have a default name but will be named however the dependent variable is named in the data frame. phi represents the precision of the beta distribution, zoi is the zero-one inflation, i.e., the probability of zero or one, and coi is the conditional one inflation, i.e., the probability of one given a zero or a one. Here, we specify our sole predictor, group, together with an intercept, $1+$, on all four parameters, such that group can influence all parts of the model, but in principle one could add either fewer or additional predictor terms to one or more of the parameters. bf () facilitates the specification of several sub-models within the same formula argument. We then pass sim_dat to the

\footnotetext{
${ }^{5}$ Parts of the code for this section is modified with permission after Vuorre (2019).
} 
Table 1

Parameter estimates of 'sim_mod' after applying inverse link functions to posterior draws.

\begin{tabular}{lllll}
\hline Parameter & Estimate & Est.Error & Q2.5 & Q97.5 \\
\hline b_Intercept & 0.26 & 0.02 & 0.23 & 0.30 \\
b_phi_Intercept & 4.41 & 0.53 & 3.42 & 5.51 \\
b_zoi_Intercept & 0.53 & 0.03 & 0.47 & 0.59 \\
b_coi_Intercept & 0.65 & 0.04 & 0.57 & 0.73 \\
b_groupB & 0.69 & 0.02 & 0.64 & 0.74 \\
b_phi_groupB & 0.73 & 0.03 & 0.67 & 0.79 \\
b_zoi_groupB & 0.42 & 0.04 & 0.34 & 0.50 \\
b_coi_groupB & 0.67 & 0.07 & 0.53 & 0.79 \\
\hline
\end{tabular}

Note. Point estimates are posterior means.

data argument and set the model family to the zero-one inflated beta distribution, which is native to brms. Implicit in the zero-one inflated beta distribution are the link functions, $\mathrm{mu}=$ logit; phi = log; zoi = logit; coi = logit, which allows the fitting of linear models to non-linear relationships. They are important to keep in mind when inspecting the output. All of this is wrapped within brm(), the fitting command of the brms package. The output (not shown directly here) can be summarized with summary(sim_mod).

In the summary output, Population-Level Effects holds the model estimates alongside some chain diagnostics, which appear acceptable (a thorough discussion of diagnostic metrics of Bayesian models is outside the scope of this tutorial but see e.g., Gelman et al., 2013; Kruschke, 2014; McElreath, 2020). To get the estimates on their original scales, however, we need to apply their inverse link functions. Since mu, zoi, and coi are all in log odds, the logistic function will transform these estimates to their original scales, namely the mean of the beta distribution and the probability of zero and/or one, respectively, whereas the phi parameters need to be exponentiated due to the applied log link. Importantly, these transformations should be applied to draws from the posterior distribution and not on the estimates provided by the summary() function, which would amount to summarizing a summary, in turn yielding incorrect results. Table 1 reports the estimates on their original scales.

The _Intercept coefficients are the intercepts for each of the sub-models (i.e., the estimates for group A), while the _groupB terms are the differences between group A and B for each sub-model. A cursory inspection reveals group to have a positive association with both the mean of the beta distribution groupB and the conditional one-inflation coi_groupB in that both estimates and their intervals are solidly above 0.5 .

As is often the case with generalized linear models and in particular in mixture models, model results are easier to inspect when plotted. Figure 1 plots the raw data points (light and dark blue, slightly jittered to prevent over-plotting) and densities for each group together with model predictions of expected values within a $95 \%$ interval, taking into account all parameters excluding phi. It appears from the distribution of raw data that group B has fewer zeros, a higher mean, and more ones, which is consistent with the model predicting 


\footnotetext{
${ }^{6}$ When assessing the difference between groups, it's generally considered best practice to compute the contrast in expected values between the groups instead of simply comparing their respective intervals of expected values; see e.g., McElreath (2020), p. 331.
} estimates ${ }^{6}$.

All in all, given sampling and simulation error with a finite sample as well as the joint nature of the sub-models, these results recover the parameters with which the data were simulated. Specifically, we simulated the intercepts as zoi $=0.5$, coi $=0.65, \mathrm{mu}=$ 0.25 , and phi $=5$, and group $\mathrm{B}$ to have a 0.2 positive difference compared to group $\mathrm{A}$ on both the mean of the beta distribution and the conditional one inflation, but no association between group and the precision or the zero-inflation.

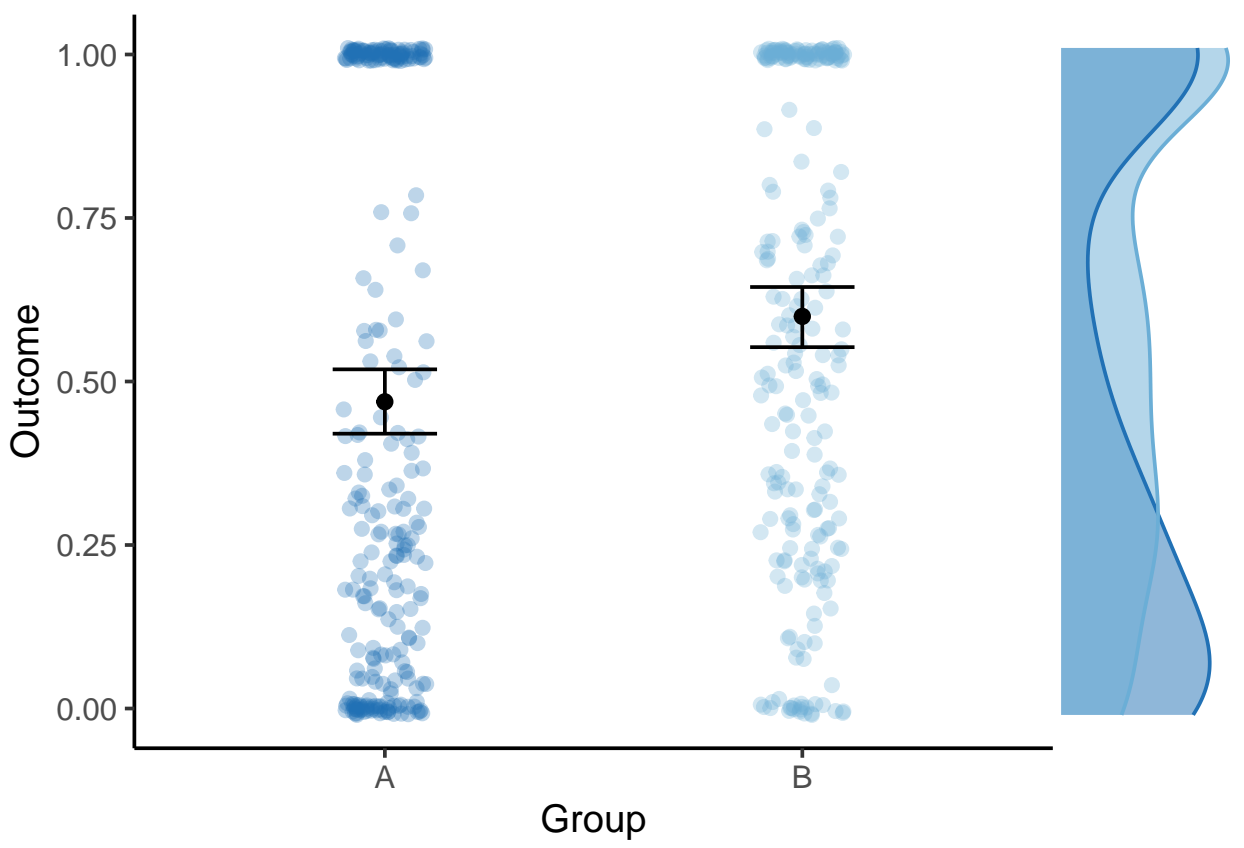

Figure 1. Plot of simulated data and model predictions of expected values. Raw data points (light and dark blue, slightly jittered) and densities from the two simulated groups. Posterior medians (black points) with $95 \%$ credible intervals.

Finally, we can compare the fit of the ZOIB to simulated data with two alternative likelihood models, the Gaussian distribution and the transformed beta (cf., discussion above). Figure 2 plots the marginal distribution of simulated outcome data (dark blue line) against 50 posterior predictive draws (light blue lines) from the three different likelihood models fitted to the simulated data. The $x$-axis represents the possible range of outcome values and the $y$-axis represents the density of each outcome value. Ideally, the predictive draws should show reasonable resemblance with the observed data. The ZOIB clearly exhibits the best fit of the three models, with the Gaussian over-shooting the mean of the simulated data but under-shooting the number of zeros and ones, and the transformed beta in turn under-shooting the mean, over-shooting extreme values and, critically, drops off just before

higher expected values on average in group B, supporting our inspection of the posterior 
zero and one.



Transformed beta

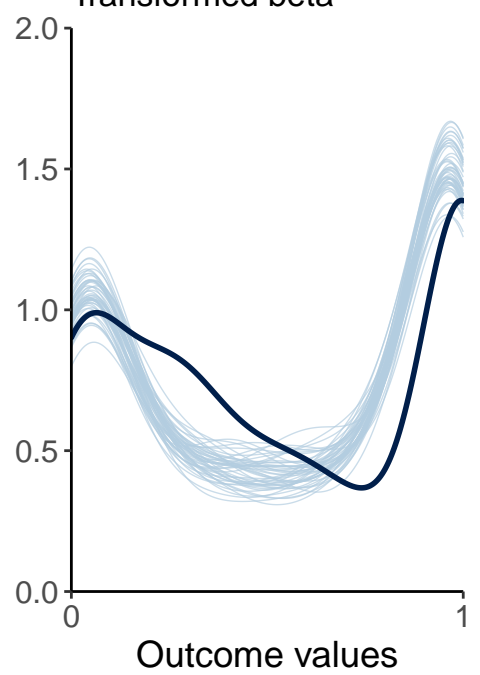

Zero-one inflated beta

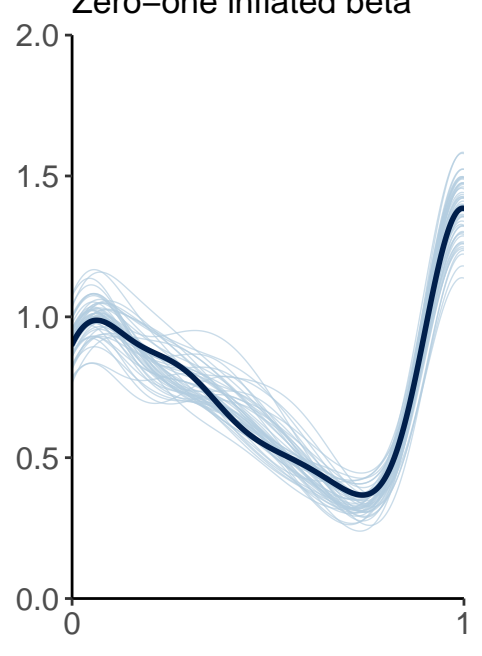

Figure 2. Marginal distribution of simulated outcome data (dark blue lines) against posterior predictive draws (light blue lines) from three different likelihood models fitted to the simulated data.

1.3.2 Discussion. The previous section illustrated the basic implementation and utility of the ZOIB in the context of zero-one inflated and bounded data, such as free-list salience scores. However, by no means have we demonstrated that the ZOIB is superior to all alternatives under all circumstances. For instance, our walk-through was based on a single simulation run with a fixed set of parameters yielding a somewhat unusual distributional shape. It is not surprising that the ZOIB exhibited the best fit since, after all, the data were simulated from a ZOIB distribution! Our goal here is simply to demonstrate that the ZOIB is a viable option to consider in the context of free-list data and similar zero-one inflated and bounded data and to provide readers with some readily-implementable computational tools. With that said, there are limitations to the ZOIB, including the risk of over-parameterization and, thereby, over-fitting (Kubinec, 2022). Similarly, it is unclear whether the ZOIB plausibly reflects any specific cognitive data-generating processes. For instance, in a free-list task, are the cognitive processes that give rise to zeros and/or ones distinct from those giving rise to intermediate values, as modeled with a ZOIB?

In this tutorial, we focus on modeling free-list data in the form of salience scores, as they are a popular transformation of free-list data that simultaneously takes into account both the position of a target item and the total number of items listed per participant while retaining an intuitive interpretation. However, it is of course possible to model free-list data more directly, as for instance modeling the presence/absence of a target item. This is straightforward with a logistic (Bernoulli) regression. Further, using a binomial distribution, researchers can model the number of times a participant lists certain items of interest (i.e., the number of "sucesses") given the total number of items listed (i.e., the number of "trials"), which in turn can be allowed to vary across participants. Alternatively, researchers could 


\footnotetext{
${ }^{7}$ Thanks to Matti Vuorre for this suggestion, as well as for a general comment on keeping the datagenerating process in mind when modeling free-list data.

${ }^{8}$ Thanks to an anonymous reviewer for this suggestion.

${ }^{9}$ All of the considered models - as well as the model families of which they form a small part - are off-the-shelf options that are increasingly available in contemporary statistical software, and they translate into a very wide range of powerful, practical applications. Eventually, however, analysts could aim to build more "bespoke" models of free-list data, that is, models that are catered to the actual assumed cognitive processes at play. As such models by their very nature sacrifice generalizability for context-specificity, they are necessarily outside the scope of the present tutorial.
} 
Table 2

Overview of field sites

\begin{tabular}{llll}
\hline Site & \multicolumn{1}{c}{ Main Economy } & Moralistic Deity & \multicolumn{1}{c}{ Local Deity } \\
\hline Coastal Tanna, Vanuatu & Horticulture/Hunting & Christian God & Garden Spirit (Tupunus) \\
Inland Tanna, Vanuatu & Horticulture/Hunting & Kalpapen & Garden Spirit (Tupunus) \\
Lovu, Fiji & Wage Labor & Hindu Bhagwan & - \\
Mauritius & Wage Labor/Farming & Hindu Shiva & Spirit/Soul/Ghost (Nam) \\
Pesqueiro, Brazil & Wage Labor & Christian God & Virgin Mary \\
Tyva Republic & Wage Labor/Herding & Buddha-Burgan & Spirit-Masters (Cher eezi) \\
Yasawa, Fiji & Fishing/Farming & Christian God & Ancestor Spirits (Kalou-vu) \\
\hline
\end{tabular}

methodological explanations for the null result and highlight key implications for the study of moralizing religious traditions. Finally, we argue more generally that the free-list method has broad potential across the social sciences with regards to how researchers measure and model individual-level and cross-cultural variation in mental representations (e.g., beliefs, attitudes, values). For the sake of the tutorial, we again integrate code in the text. The following sections contribute to the tutorial by demonstrating a practical implementation of the ZOIB in a more realistic data setting.

\section{Methods}

\subsection{Data}

The present data are publicly available and originate from The Evolution of Religion and Morality Project ${ }^{10}$. The main study consisted of a battery of demographic and religiosity questions as well as behavioral economic games (for further methodological details and presentations of field sites, see Bendixen, Apicella, et al., forthcoming; Lang et al., 2019; Purzycki et al., 2016b, 2016a; Purzycki, Henrich, et al., 2018). Specifically for present purposes, participants were asked about the concerns of two different locally relevant deities (a "moralistic" deity and a "local" deity) using both a three-item scale and a free-list task. Table 2 provides an overview of the field sites, their main economies, and the selected deities. For site-specific research reports, see Tanna, Vanuatu: Atkinson (2018); Lovu, Fiji: Willard (2018); Mauritius: Xygalatas et al. (2018); Pesqueiro, Brazil: Cohen, Baimel, and Purzycki (2018); Tyva Republic: Purzycki and Kulundary (2018); Yasawa, Fiji: McNamara and Henrich (2018).

\subsection{Moral interest scale}

The moral interest scale took the form: How important is it for [moralistic/local deity] to punish: [theft/lying/murder]? Responses were on scales of 0 to 4: (0) Not important at all; (1) A little important; (2) Important; (3) Very important; and (4) The most important. Responses are transformed into an index scale ranging from 0 to 1 , by dividing each participant's score on the items by the maximum possible score that each participant could

\footnotetext{
${ }^{10}$ Full datasets and protocols for the greater project are publicly available at: https://github.com/bgpurzy cki/Evolution-of-Religion-and-Morality.
} 


\footnotetext{
${ }^{11}$ One issue with this approach is that it assumes that, in cases of missing responses, participants are consistent across the three items - e.g., a participant can get a 1 on the index either by selecting (4) on all three items or on just one or two of the items, if failing to respond to the remaining item(s). Another issue is the lack of uncertainty around the index score. There are other - and arguably more principled-ways of handling missing values, such as full Bayesian imputation (e.g., Erler et al., 2016; McElreath, 2020). We refrain from pursuing this here for a couple of reasons. First, this particular scale has been used in previous research (Lang et al., 2019; Purzycki et al., 2016b) where it failed to predict behavioral outcome in economic games, and so we were interested in associating it with an additional instrument, the free-list task. Second, handling missing values in item scales is outside the scope of this tutorial.
} 
the Morality free-list code and their overall response on the moral interest scale for the given deity. There are many ways to go about this - see source code for one example. We can check the structure str() of the resulting data frame, where id is participants' ID numbers, $\mathrm{y}$ is salience score for free-listed Morality, culture is cultural group, and scale is overall response on the moral interest scale for the moralistic god's moral concerns.

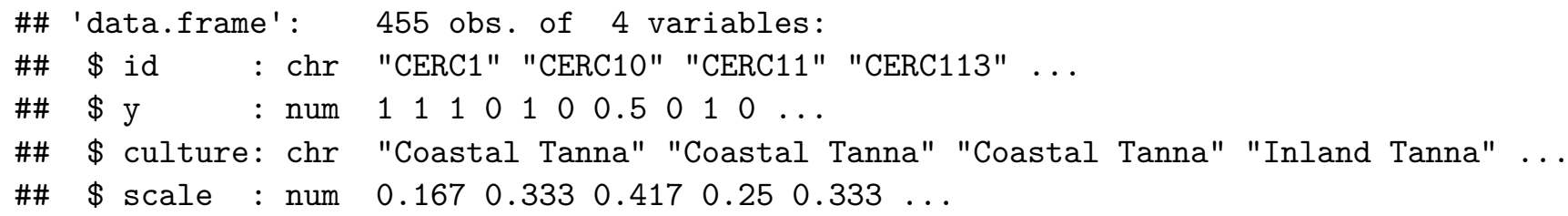

\section{Modeling salience scores}

Recall that we want to assess whether the moral interest scale of a focal deity corresponds to salience of moral items in the free-list task. Specifically, if a participant responded on the scale that a focal deity (moralistic or local deity) was morally punitive, both overall on the scale and on sub-items (theft, lying, murder), would corresponding responses - that is, "Morality" as a general code and, as specific codes, theft, lying and murder - also be higher up on a participant's free-list regarding what angers the focal deity? We construct a series of models assessing these relationships: 1) for both the moralistic and local deity, 2) between the index score on the scale and free-listed "Morality" (general code), and 3) between each item of the scale (theft, lying, murder) and the salience of each of these focal items in the free-lists. Since we have no reason to suspect systematic missingness patterns, we perform complete-case analysis. Below, we detail our analysis, first in formal notation and then in $\mathrm{R}$ code.

\subsection{Overview}

We model two deities (moralistic and local) and for each of these we model, first, the relationship between free-listed, general-coded "Morality" and the moral interest scale index (Model 1 and Model 2) and then the relationship between free-listed, specifically-coded moral items (theft, lying, murder) and the corresponding single items of the moral interest scale (Models 3-8). This yields eight models. As we cluster by field site, these are multilevel models, and for each main model, we also fit a corresponding "null" model that excludes scale response as a predictor of the free-list outcome but retains varying effects of field site, and then employ approximate leave-one-out cross-validation (Vehtari et al., 2020; Vehtari, Gelman, \& Gabry, 2017) to assess the relative out-of-sample predictive performance of models with and without scale as a predictor. Throughout model-building, checking and inference, we informally follow a Bayesian workflow (Gelman et al., 2020). In the following sections, we spell out the general formal form of the eight main models, starting with Models $1-2$, as well as their implementation in $\mathrm{R}$. 


\subsection{Formal form of Models 1-2: Predicting salience of general Morality in the free-list task}

In order to predict the individual-level salience of moral items $Y_{i}$, all linear models $(\mu, \phi, \alpha, \gamma)$ include: a global intercept $\beta^{\text {Intercept }}$, site-specific intercepts $\beta_{\operatorname{SITE}[i]}^{\text {Interept }}$, a global slope for scale score $\beta^{\text {Scale }} S_{i}$, and varying slopes across sites for scale score $\beta_{\operatorname{SITE}[i]}^{\text {Scale }} S_{i}$. The joint ZOIB model thus takes the general formal form:

$$
\begin{aligned}
Y_{i} & \sim \operatorname{ZOIBeta}\left(\mu_{i}, \phi_{i}, \alpha_{i}, \gamma_{i}\right) \\
\operatorname{logit}\left(\mu_{i}\right) & =\beta_{\mu}^{\text {Intercept }}+\beta_{\mu, \operatorname{SITE}[i]}^{\text {Intercet }}+\beta_{\mu}^{\text {Scale }} S_{i}+\beta_{\mu, \text { SITE }[i]}^{\text {Scale }} S_{i} \\
\log \left(\phi_{i}\right) & =\beta_{\phi}^{\text {Intercept }}+\beta_{\phi, \text { SITE }[i]}^{\text {Intercet }}+\beta_{\phi}^{\text {Scale }} S_{i}+\beta_{\phi, \text { SITE }[i]}^{\text {Scale }} S_{i} \\
\operatorname{logit}\left(\alpha_{i}\right) & =\beta_{\alpha}^{\text {Intercept }}+\beta_{\alpha, \text { SITe }[i]}^{\text {Intercet }}+\beta_{\alpha}^{\text {Scale }} S_{i}+\beta_{\alpha, \text { SITE }[i]}^{\text {Scale }} S_{i} \\
\operatorname{logit}\left(\gamma_{i}\right) & =\beta_{\gamma}^{\text {Intercept }}+\beta_{\gamma, \text { SITE }[i]}^{\text {Intercet }}+\beta_{\gamma}^{\text {Scale }} S_{i}+\beta_{\gamma, \text { SITE }[i]}^{\text {Scale }} S_{i}
\end{aligned}
$$

where $\mu$ and $\phi$ represent, respectively, the mean and the precision of the beta distribution, $\alpha$ represents "zero-one inflation" (the probability that an observation is either 0 or 1 ) and $\gamma$ represents "conditional one inflation" (the probability that, given an observation is 0 or 1 , the observation is 1 ).

We define our priors as follows:

$$
\begin{aligned}
\beta_{\mu}^{\text {Intercept }} & \sim \operatorname{Normal}(0,1) \\
\beta_{\mu}^{\text {Scale }} & \sim \operatorname{Normal}(0,2.5) \\
\beta_{\phi}^{\text {Intercept }} & \sim \operatorname{Normal}(2,0.5) \\
\beta_{\phi}^{\text {Scale }} & \sim \operatorname{Normal}(-1,1) \\
\beta_{\alpha}^{\text {Intercept }} & \sim \operatorname{Normal}(0,1) \\
\beta_{\alpha}^{\text {Scale }} & \sim \operatorname{Normal}(0,2.5) \\
\beta_{\gamma}^{\text {Intercept }} & \sim \operatorname{Normal}(0,1) \\
\beta_{\gamma}^{\text {Scale }} & \sim \operatorname{Normal}(0,2.5)
\end{aligned}
$$

We arrived at these priors through a combination of formal reasoning and iterative cycles of prior predictive checks (see below for further details). In brief, prior predictive checks are simulations from the generative model before fitting the model to data. That is, what kinds of relationships is the model "expecting" prior to the data.

Our priors are meant to be weakly regularizing such that they constrain the estimates to be mostly within realistic ranges and are mildly skeptical toward strong statistical relationships. It is important to stress, however, that priors should be interpreted in the context of the data and model of which they are part (Gelman, Simpson, \& Betancourt, 2017), and that these priors might therefore not generalize to other modeling scenarios. 
Developing principled and practical approaches to eliciting appropriate prior distributions is an active research area, and further discussion is outside the scope of this tutorial (for a technical review, see Mikkola et al., 2021; for a practical introduction, see McElreath, 2020).

Except for $\phi$, the coefficients are on the log odds scale, meaning that to get the coefficients on their original scale, we first turn them into odds by exponentiating them (see Part One). The original scale is then odds/(1 + odds $)$. So, for instance, a Gaussian prior on $\beta_{\mu}^{\text {Intercept }}$ with mean 0 on the log odds scale means that the coefficient is centered on $\exp (0) /(\exp (0)+1)=1 /(1+1)=0.5$, which is the midpoint of our outcome variable (i.e., saliences scores ranging from 0 to 1 ). Likewise, since two standard deviations roughly cover $95 \%$ probability of a normal distribution, assigning a prior of $\operatorname{Normal}(0,1)$ sets the bulk of the prior probability mass to be within the range of $\exp ( \pm 2) /(\exp ( \pm 2)+1)$ $\approx \pm 7.38 /( \pm 7.38+1) \approx[0.12,0.88]$.

In this particular parameterization, the $\beta^{\text {Scale }}$ coefficients represent the estimated change in outcome (i.e., salience score) going from zero on the predictor (i.e., the lowest response on the index) to 1 (i.e., the maximum response on the index). A $\operatorname{Normal}(0,2.5)$ prior is therefore sensible in that it puts some probability mass on the entire range between $\approx-1$ and $\approx 1$ while being centered on 0 (i.e., no change) ${ }^{12}$.

As we cluster on field site, each linear model $(\mu, \phi, \alpha, \gamma)$ has its own multi-level structure, which is formally defined as follows. The varying effects across sites are bound in a variance-covariance matrix with multivariate priors of a Gaussian variety (with means of 0 as these are already included in the linear models above):

$$
\left[\begin{array}{l}
\beta_{\mu, \phi, \alpha, \gamma, \text { SITE }}^{\text {Intercet }} \\
\beta_{\mu, \phi, \alpha, \gamma, \text { SITE }}^{\text {Scale }}
\end{array}\right] \sim \text { Multivariate Normal }\left(\left[\begin{array}{l}
0 \\
0
\end{array}\right], \text { SRS }\right)
$$

Here, $\mathbf{S}$ is a diagonal matrix of intercept and predictors' standard deviations, $\sigma_{p}$,

$$
\begin{aligned}
\mathbf{S} & =\left[\begin{array}{cc}
\sigma_{\beta_{\mu, \phi, \alpha, \gamma}^{\text {Intercept }}} & 0 \\
0 & \sigma_{\beta_{\mu, \phi, \alpha, \gamma}^{\text {Scale }}}
\end{array}\right] \\
\mathbf{R} & \sim \operatorname{LKJ} \operatorname{corr}(4) \\
\sigma_{p} & \sim \operatorname{Exponential}(1)
\end{aligned}
$$

while $\mathbf{R}$ is their correlation matrix with a prior distribution from the Lewandowski family (Lewandowski, Kurowicka, \& Joe, 2009).

\subsection{Implementation in $\mathrm{R}$}

All of this is implemented in R with brms as follows, illustrated with Model 1 (m1), which predicts the salience of "Morality" as a general code using the three-item moral interest scale: First, we specify our model formula. In the present study, the model formula is relatively simple, in that we are predicting salience scores (y) according to an intercept 1

\footnotetext{
${ }^{12}$ Note that when we model these priors in a joint distribution, the prior for the intercepts in the $\mu, \alpha$, and $\gamma$ sub-models widens so as to allow for the steep slopes implied by the prior on $\beta^{\text {Scale }}$.
} 
and a single predictor, the moral interest scale (scale), which is clustered according to field site (culture), yielding the basic syntax y $\sim 1+$ scale $+(1+$ scale $\mid$ culture). This specification allows each field site to have varying intercepts and slopes. We specify this structure on all four sub-models.

Next, since this is a Bayesian model, we want to explicitly set the prior distributions for each of the model parameters and hyper-parameters (see above). brms includes a convenience call, get_prior(), that returns a complete list of model parameters and their names given a formula, a data frame data, and a likelihood function family.

Then, we manually specify our priors, using the set_prior() command in brms and store it in a new object, priors_main. Note that this step is not strictly necessary to make the model run. In the absence of user-specified prior distributions, brms implements default "flat" priors that will likely work well in many applied cases and yield results that approximate maximum likelihood estimation.

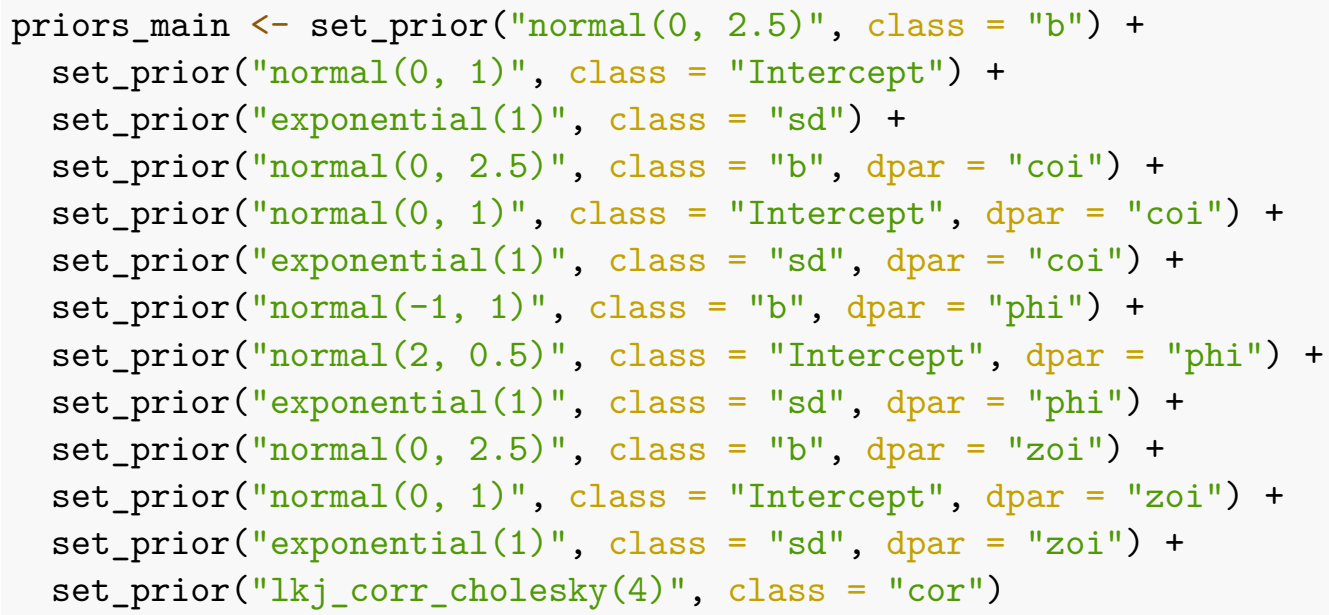

Next, we verify that these priors are sensible. We aim for so-called weakly regularizing priors, that is, priors that put most of their probability mass within realistic ranges. To do so, we perform prior predictive checks, whereby we sample from the model and prior without fitting the model to the data. Note that we now pass our formula, data, and likelihood to brm(), and that we also add our priors via the prior argument. We set sample_prior $=$ "only" so that we only sample from the prior and not the data, and a seed for reproducibility.

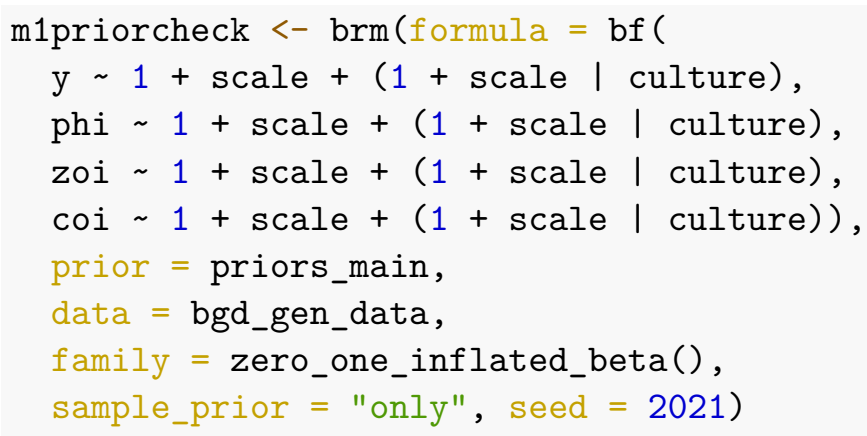


There are many options for assessing the implications of the priors (see e.g., Gabry et al., 2019; Kruschke, 2014; McElreath, 2020) and oftentimes, prior predictive checks need to be catered to the specific data and model(s) at hand (Gelman et al., 2020). Here, we show just two different readily-available visualization options. First, we can sample from the prior predictive distribution and compare those values to the empirical distribution of observed values, using the convenience function pp_check() in the bayesplot package (Figure 3A). We simply pass our prior model fit m1priorcheck to pp_check() and set the number of draws (say, 30) that we want from the prior predictive distribution ndraws $=30$, as well as a seed for reproducibility. This is essentially the same procedure as we used to generate Figure 2.

The dark blue line in the upper panel of Figure 3A is the distribution of observed values, whereas the light blue lines are draws from the prior predictive distribution, which are not yet informed by the observed data. As can be seen, the prior draws mostly fall within realistic estimates (around the observed values) and only a few draws are in extreme ranges (i.e., large numbers of zeros and/or ones). Recall that this is so even before the model has "seen" the data (the sample_prior = "only" call makes the model sample only from the priors). Note, however, that we include the observed distribution only for illustration here; ideally, the priors should be sensible a priori, that is before either the model or the analyst has inspected - or even collected - the data.

While this kernel density plot is a quick way to visualize a model's prior expectation of the marginal outcome distribution, it gives us little in the way of assessing the priors for the influence of the predictor terms; that is, what the model expects the relationship to be between the independent and the dependent variable(s). For that, we can instead plot draws from the prior predictive distribution of the outcome as a function of the predictor (Figure 3 , bottom). Each line is a regression line of expected values. As can be seen, some lines are flat, some slope downwards and yet others upwards, suggesting that the model allows a wide range of statistical relationships before having been informed by data.

Finally, we are ready to fit the full model and then perform posterior predictive checks, to assess how much the model learned from the data (Figure 3B, compared to the prior predictive checks in Figure 3A). In the following code block, the formula, data, prior, and family calls are similar to above, and we then add some explicit settings to the sampler, which in turn facilitate reasonable chain convergence and model comparison.

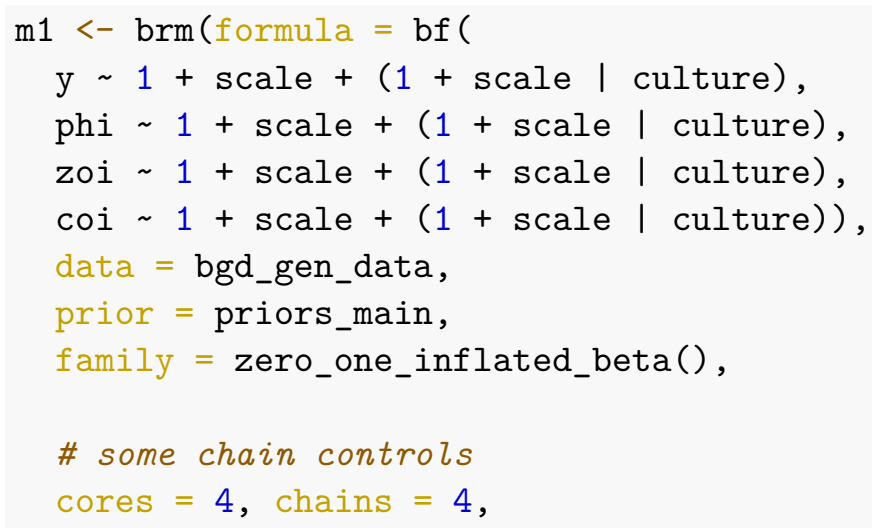





Figure 3. Predictive checks. Top: Prior (A) and posterior (B) predictions of the marginal distribution of salience scores (kernel density). The dark blue line is the distribution of observed values for Model 1. The light blue lines are draws from, respectively, the prior and posterior predictive distributions. The $y$-axis represents density, while the $x$-axis denotes salience scores. Bottom: Prior predictions for each field site from Model 1 on the relationship between the moral interest scale ( $x$-axis) and salience scores of the free-listed general Morality code ( $y$-axis). Lines are draws of expected values from the prior predictive distribution. 


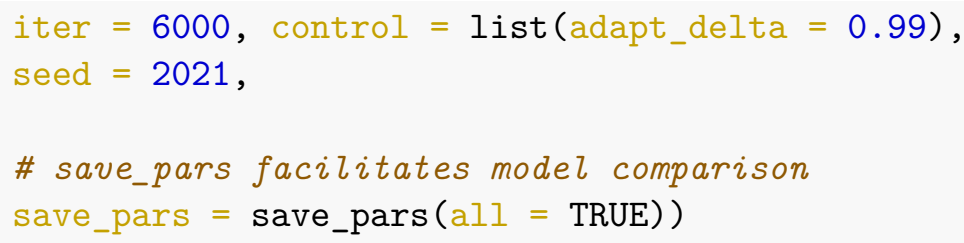

Note that the posterior predictive draws in the kernel density plot (Figure 3B) are much closer to the distribution of observed values, indicating that the model indeed has learned from the data. With summary (m1) we can get an overview of the main parameter estimates and chain diagnostics of the model (not shown here).

\subsection{Formal form and implementation of Models 3-8: Predicting specific moral items in the free-list task}

The key difference between the general-coded "Morality" models outlined in the previous section (Models 1-2) and the specific moral item models (Models 3-8) is that in the latter set we model the predictor, the single moral scale item, with a monotonic function, since it is an ordered category of response options (Bürkner \& Charpentier, 2020; Bürkner \& Vuorre, 2019; Liddell \& Kruschke, 2018). This allows each response option (from 0 to 4) to have its own conditional effect on the outcome, which is now salience of a single free-listed item. These monotonic predictors are modeled with a weakly informative prior in the form of a Dirichlet distribution, Dir $(2,2,2,2)$. This prior encodes the expectation that any of the response options could be more or less likely than the others (McElreath, 2020, pp. 391-396).

The remaining priors are otherwise identical to Models 1-2 with the following exception. Compared to Models 1-2, where the $\beta^{\text {Scale }}$ coefficients for the $\mu, \alpha$, and $\gamma$ sub-models are interpreted as the estimated change in outcome when the predictor is set at one (i.e., the maximal response on the index) compared to zero (i.e., the lowest response on the index), in Models 3-8 the $\beta$ coefficients instead represent the estimated average difference between two adjacent categorical response options (see Bürkner \& Charpentier, 2020). Therefore, we set a prior on the $\beta^{\text {Scale }}$ coefficients for the $\mu, \alpha$, and $\gamma$ sub-models in Models 3-8 to $\operatorname{Normal}(0,0.625)$, where the standard deviation of 2.5 from the Models $1-2 \beta$ priors the $\mu$, $\alpha$, and $\gamma$ sub-models is divided with 4 , the number of response options minus 1 .

To implement these changes in brms, we need only a few modifications to the $\mathrm{R}$ code above. To specify monotonicity, we simply wrap the predictor, scale, in mo() and set a Dirichlet prior on the monotonic predictors similarly to above, which we add to the prior argument (for further details, see Bürkner \& Charpentier, 2020): set_prior("dirichlet $(2,2,2,2)$ ", class="simo", dpar=c("", "coi", "phi", "zoi"), coef="moscale1").

\section{Results and Discussion}

\subsection{Results}

Here we report key results. First, we assess the posterior predictions of Model 1 and 2 (see Supplementary Section C for similar plots of Models 3-8), and then compare model comparison metrics between all models and their respective "null" models. 
Figure 4 plots, for each field site and each deity (top: moralistic deities, bottom: local deities), draws of expected values from the posterior predictive distribution ${ }^{13}$, as well as raw data points with slight jitter (compare these plots to the prior predictive plot in Figure 3, bottom). For the moralistic deities, in most sites the draw lines are mostly flat, suggesting that the relationship between the moral interest scale ( $x$-axis) and salience of the free-listed general Morality code ( $y$-axis) is weak or non-existent. At all sites for the local deities, the relationship seems to trend slightly upwards, although the estimates are quite uncertain, as vizualized by the widely-spaced draw lines at several sites. Note that we get posterior predictions for Lovu for the local deities even though we do not have data for a local deity at that particular field site. Rather, the model predicts expected values for a hypothetical unobserved field site, which we label Lovu, informed by all other sites ${ }^{14}$.

To put these results into perspective, consider the raw data points. Data points in the top-left corner of a panel are individuals who, when asked on the scale how important it is for the target deity to punish theft, lying, and murder, respond "not at all important", but free-list a moral item as the top thing that angers the target deity. And vice versa for the bottom-right corner: here, participants respond that theft, lying, and murder are all the most important thing for the target deity to punish ${ }^{15}$ but does not free-list a moral item among that deity's concerns. That a noticeable number of participants gravitated toward such response patterns is an illustration of the model-driven inferences, namely that these data and model predictions suggest no relationship - and at best a very weak and uncertain one-between the scale and the free-list task. Results are qualitatively similar when we assess the posterior predictions of the specific codes and the corresponding moral scale items (see Supplementary Section C).

This interpretation is further bolstered when we compare each main model against its respective "null" model. Table 3 reports raw (i.e., without having applied their inverse link functions, unlike in the simulated case above) $\beta$ coefficients (posterior means) with 95\% credible intervals for each main model's focal parameters. If the scale and the free-list task is positively associated, we would expect either of these parameters to have estimates where the bulk of the interval is above zero (with the caveat that the one inflation parameter $\beta_{\gamma}^{\text {Scale }}$ is conditional on the zero-one inflation parameter $\left.\beta_{\alpha}^{\text {Scale }}\right)$. Table 3 also reports model comparison metrics obtained with approximate leave-one-out cross-validation (LOO-CV). In a multilevel context, LOO-CV is an indicator of a model's accuracy in predicting a new observation in one of the observed groups ${ }^{16}$. LOO-CV is a preferable model comparison

\footnotetext{
${ }^{13}$ More formally, these are draws from the posterior distribution given by: $\mathrm{E}[y]=\operatorname{Pr}($ zero or one $) \times \operatorname{Pr}($ conditional one $)+$ mean of the beta distribution $\times \operatorname{Pr}($ neither zero nor one $)=$ $\alpha \times \gamma+\mu \times(1-\alpha)$ (see Ospina \& Ferrari, 2012). As can be seen, this calculation ignores the precision parameter of the beta distribution $\phi$.

${ }^{14}$ Specifically, expected values for Lovu are sampled from "the (multivariate) normal distribution implied by the group-level standard deviations and correlations" (see prepare_predictions.brmsfit documentation)

${ }^{15}$ For present purposes, we ignore the logical incoherence of such a response pattern (i.e., responding that two or more distinct behaviors are the most important thing to punish).

${ }^{16}$ An alternative approach is leave-one-group out cross-validation (LOGO-CV) which assesses a model's accuracy in predicting a new observation in a new (unobserved) group. This procedure is more computationally intensive, because it involves refitting the model $k$ times, where $k$ equals the number of observed groups. See Supplementary Section C for an implementation. Results are qualitatively similar to LOO-CV in the present study.
} 


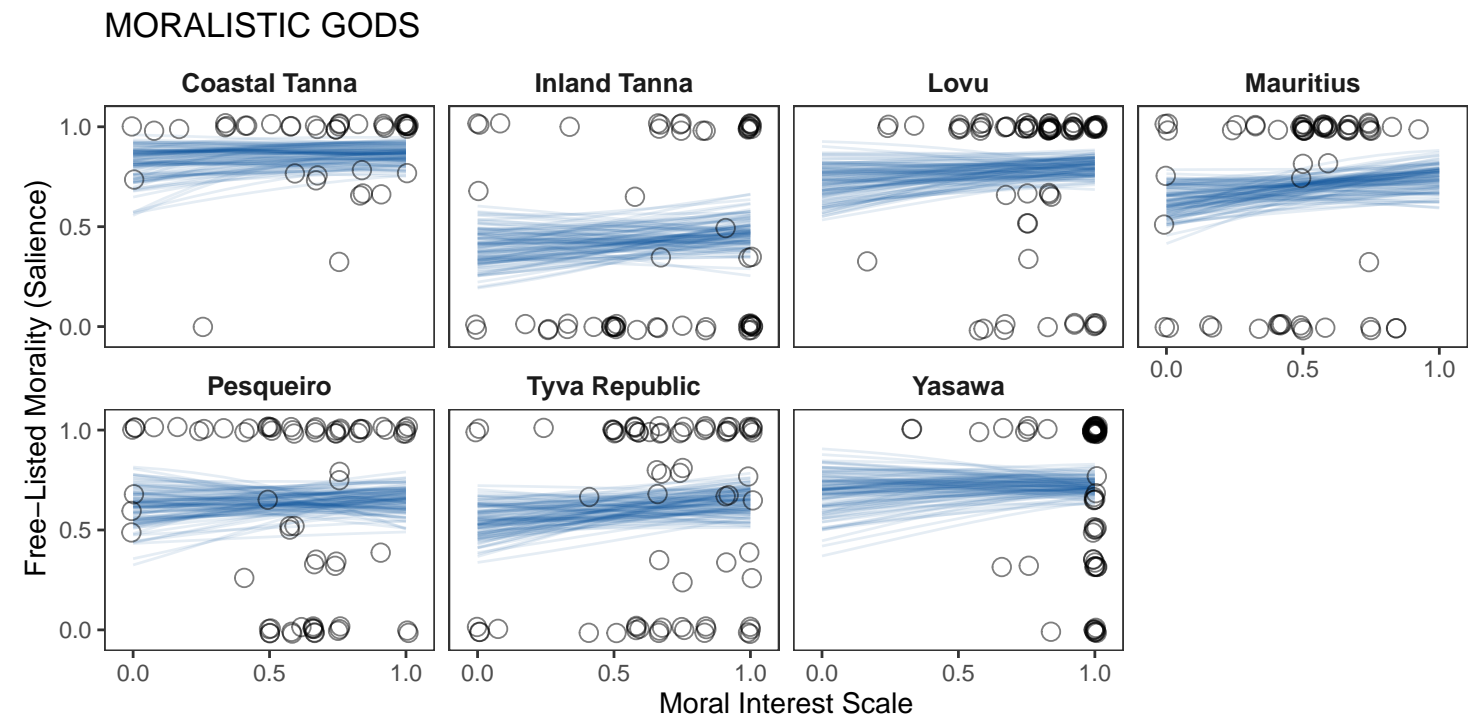

LOCAL GODS

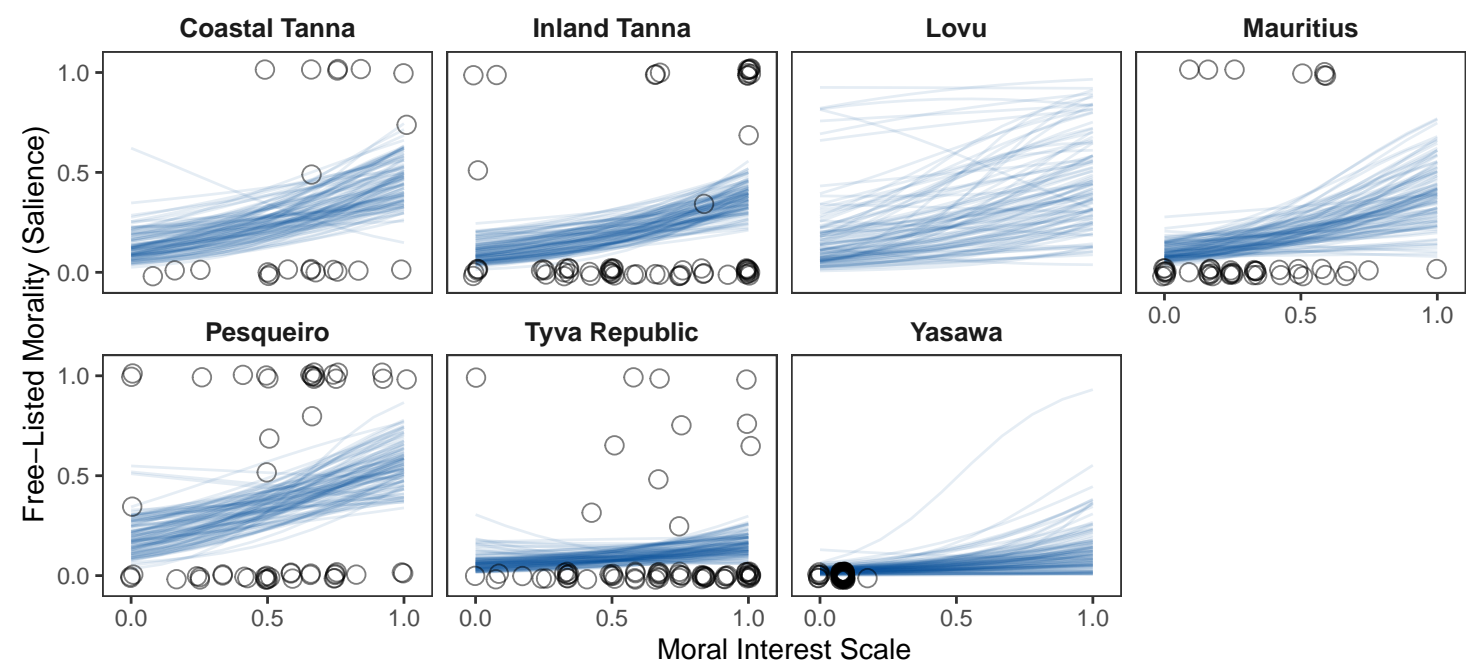

Figure 4. Posterior predictions for each field site and each deity from Model 1 and 2 on the relationship between the moral interest scale ( $x$-axis) and salience of the free-listed general Morality code ( $y$-axis). Lines are draws of expected values from the posterior distribution. Raw data points are slightly jittered. 
metric as it penalizes model complexity. This is desirable for present purposes as we aim to identify the best performing and most parsimonious model within each of the eight model pairs. Akaike weights are calculated on the basis of the leave-one-out information criterion and a higher value means more weight, i.e. the model that the algorithm more heavily favors. Expected log posterior density (ELPD) is a measure of overall model fit and out-of-sample predictive accuracy. Here, we report the ELPD difference $\delta$ and the standard error (SE) of the difference between the best performing model, denoted by an asterisk, and the remaining model in the pair.

While ELPD differences are generally small compared to their standard errors, in most cases we find that LOO-CV either favors the null models or favors none of the models in particular, indicating that including scale response as a predictor of moral salience in the free-list task does not generally improve out-of-sample predictive performance when penalizing increased model complexity. The only clear exception to this pattern is the Model 5 pair, where the full model (m5) is clearly favored over the null model. Note, however, for this particular model, we had chain convergence issues, potentially rendering estimates unreliable. Further, in an alternative specification of the Model 5-pair using the ordered beta distribution as the outcome likelihood the interval of the $\beta$ coefficient straddles zero, although here too LOO-CV favors the main model over the null (see Supplementary Section A). However, upon inspecting field-site specific estimates this appears primarily driven by the Lovu data (see Supplementary Section C). Overall, then, we infer from these lines of evidence that there is no clear and reliable association between the moral interest scale and the free-list task across deities and field sites.

\subsection{Discussion}

We now turn to a discussion of, first, possible methodological accounts of the null result, and then consider some of its key implications.

First, many participants did not free-list moral items - note the zeros along the $x$-axes in Figure 4. One potential issue might be that these zeros drive the non-association between the two instruments. However, we claim that excluding participants who did not free-list moral items in this case is misleading. These participants could have listed moral items, but they simply did not. Therefore, there is information in the zeros, which may in turn help guide interpretation. On the other hand, since the free-list task was capped at five items (a few participants did list more than that), it stands to reason that participants potentially could have listed moral items eventually but that the design did not allow for it ${ }^{17}$. Of course, items listed later would have a lower salience score and so this argument does not account for the - at best - very weak and uncertain relationship between the two instruments. To address this, we re-ran the main models $(\mathrm{m} 1-\mathrm{m} 8)$ with reduced data sets which only included non-zero free-list responses. Again, we found no reliable association between the two instruments across deities and field sites (see Supplementary Section C).

Other methodological limitations should also be considered. It could be, for instance, that the non-existing relationship between the two instruments is simply an artifact of the

\footnotetext{
${ }^{17}$ As an anonymous reviewer notes, this situation could be interpreted as censoring and as such approached with a survival model.
} 
Table 3

Focal parameter estimates for all models and model comparison metrics.

\begin{tabular}{|c|c|c|c|c|c|c|}
\hline & $\beta_{\mu}^{\text {Scale }}$ & $\beta_{\alpha}^{\text {Scale }}$ & $\beta_{\gamma}^{\text {Scale }}$ & Akaike Weights & $\delta$ ELPD $[\mathrm{SE}]$ & $N$ \\
\hline $\mathrm{m} 1$ & $-0.35[-0.98,0.29]$ & $-0.20[-1.30,0.90]$ & $0.54[-0.52,1.60]$ & 0.10 & $-1.86[2.11]$ & 455 \\
\hline m1_null & - & - & - & 0.90 & $*$ & \\
\hline $\mathrm{m} 2$ & $1.05[-0.23,2.38]$ & $-0.94[-2.75,0.86]$ & $1.79[0.34,3.31]$ & 0.76 & $*$ & 326 \\
\hline m2_null & - & - & - & 0.24 & $-1.09[3.76]$ & \\
\hline m3 & $0.03[-0.11,0.17]$ & $-0.02[-0.25,0.23]$ & $0.09[-0.26,0.42]$ & 0.02 & $-4.13[1.90]$ & 451 \\
\hline m3_null & - & - & - & 0.98 & $*$ & \\
\hline $\mathrm{m} 4$ & $-0.08[-0.34,0.18]$ & $-0.03[-0.34,0.29]$ & $-0.42[-0.95,0.10]$ & 0.01 & $-4.38[2.01]$ & 451 \\
\hline m4_null & - & - & - & 0.99 & $*$ & \\
\hline m5 & $-0.03[-0.10,0.04]$ & $0.14[-0.02,0.28]$ & $0.25[0.17,0.40]$ & 1.00 & * & 451 \\
\hline m5_null & - & - & - & 0.00 & $-37.46[18.54]$ & \\
\hline $\mathrm{m} 6$ & $-0.19[-0.74,0.33]$ & $0.07[-0.46,0.64]$ & $-0.05[-0.82,0.59]$ & 0.10 & $-2.10[1.95]$ & 326 \\
\hline m6_null & - & - & - & 0.90 & $*$ & \\
\hline $\mathrm{m} 7$ & $-0.09[-0.59,0.49]$ & $-0.07[-0.64,0.52]$ & $-0.54[-1.35,0.24]$ & 0.16 & $-1.66[1.42]$ & 326 \\
\hline m7_null & - & - & - & 0.84 & $*$ & \\
\hline $\mathrm{m} 8$ & $-0.05[-0.58,0.49]$ & $0.14[-0.45,0.77]$ & $-0.61[-1.65,0.44]$ & 0.01 & $-5.05[2.89]$ & 326 \\
\hline m8_null & - & - & - & 0.99 & & \\
\hline
\end{tabular}

Note. Point estimates of the raw $\beta$ coefficients are posterior means with $95 \%$ credible intervals in brackets. $\delta$ ELPD $=$ differences in expected log posterior density between model pairs; $\mathrm{SE}=$ standard error of ELPD differences. The best performing model of the eight pairs is denoted by an asterisk. $N$ is the total number of observations used in each model (identical across model pairs).

study protocol. Participants completed the scale before completing the free-list task. Hence, it is possible that participants were simply reluctant to free-list the target moral items (theft, murder, lying) because they had already completed scale items pertaining to these. We are skeptical of this explanation for several reasons. First, this does not explain the non-relationships for moral items in general (Models 1-2), as the Morality code includes moral behaviors in general, not only the three target moral items that were the focus in the item response scale. Second, the protocol was quite long (around 90 minutes), and the free-list task was not preceded directly by the moral interest scale. Therefore, we further deem it unlikely that the scale items had a noticeable effect on the free-list task. Third, this ordering effect cannot account for the disparate responses that participants gave across field sites and deities. Note that there are many more non-zero free-list responses in the panels of the moralistic deities compared to the local deities. In other words, if participants were reluctant to free-list moral items because they had already completed the moral items, why were they then more likely to free-list moral items for the moralistic gods than the local gods? Fourth, the ordering effects explanation contradicts another possible explanation for the results, namely that some kind of priming influenced the free-list task. Just as the order effects could hamper the tendency to report the target items, in principle it is also possible that the scale items could have increased the tendency to report the target items, perhaps through a priming mechanism. We are skeptical of the priming explanation for the same reasons as the reverse and in general. In sum, ordering effects are not plausible explanations 
in our view.

Lastly, one could object that the instructions for the two tasks are not sufficiently similar to warrant an empirical comparison; the item scale asks about behaviors that a given deity might punish whereas the free-list task probes the kinds of things that angers a given deity. We concede this objection. However, we do not think that it explains away the reported results. Ethnographically, there is often considerable overlap between what a deity is angered by and what said deity punishes, at least for these data (Bendixen, Apicella, et al., forthcoming) but also in the ethnographic record more generally (e.g., Bendixen \& Purzycki, forthcoming). And so, while we would perhaps not expect a perfect association between the two tasks, we would at least expect a detectable positive association. Of course, another possibility is that the two tasks measure distinct cognitive processes. While our data do not allow us to make inferences one way or the other, we speculate that while free-list tasks might elicit cultural recall data, scale items might prompt reasoning about spiritual agents or agents in general (Purzycki et al., 2022). These distinct cognitive modes may in turn account for inconsistencies between cultural models and scale responses (see also Purzycki, 2011) and represents an intriguing area of future research.

The reported null results speak directly to current debates in the cognitive and evolutionary sciences of religion. A rapidly growing body of work studies the relationship between moralizing religions and deities and social behavior (Bendixen, Lightner, et al., forthcoming), and item response scales are very common instruments as measures of moral salience of deities and religions. However, our finding that an item response scale on a target deity's moral concerns does not predict moral salience of that deity in a free-list task raises questions about current methodological approaches and is consistent with another recent cross-cultural study that failed to find clear evidence of an association between item scales and free-list tasks on moral and political attitudes (White \& Norenzayan, 2021).

To complicate matters further, in previous cross-cultural analyses on a data set of which the present one is a subset, the moral interest scale failed to predict individual-level behavior in two different economic games involving more than 2000 participants from 15 diverse field sites (Lang et al., 2019). This finding casts doubt on either a) the hypothesis that moral salience of a deity per se is associated with cooperative tendencies on the individual level, b) the prospect of using item response scales to capture individual-level variation relevant to this domain, or c) both. An additional downside to item response scales identified by this cross-cultural research is that Likert scales are not intuitive for some peoples relatively isolated from Western influence (Apicella, 2018; Purzycki et al., 2016a; Purzycki et al., 2022), a limitation that does not apply to oral free-listing (Quinlan, 2017).

In summary, in contrast to pre-fabricated item response scales, free-listing is an unambiguous measure of pools of cognitive and cultural information; participants drawn from the same sample independently tend to exhibit convergence with regard to their mental models, given a valid topic (see e.g., Bendixen, Apicella, et al., forthcoming; Purzycki \& Bendixen, 2020; Purzycki, Pisor, et al., 2018; Willard et al., 2020). Free-listing is also arguably a more natural analog to how people in their day-to-day lives socially express, transmit, and exchange mental representations. For these reasons, we encourage researchers interested in measuring cognitive and cultural models to consider including alongside an 


\footnotetext{
${ }^{18}$ As an anonymous reviewer notes, specifying more flexible and "wiggly" predictor terms (e.g., as in generalized additive models, Wood, 2017) is of course also a possibility, if motivated by appropriate theory or context (e.g., time series).

${ }^{19}$ By "ethnographic" we mean simply "the processes and products of research that document what people know, feel, and do in a way that situates those phenomena at specific times in the history of individual lives" (Handwerker, 2001, p. 7).
}

item response scale a corresponding free-list task so as to ensure that their scales have explanatory power above and beyond a measure of task-specific item response behavior.

\section{General Discussion and Conclusion}

Here, we have 1) offered a tutorial on how to prepare and statistically model salience scores from a free-list task in Bayesian regression with various predictor terms (i.e., a binary predictor in Part One and a continuous and a categorical predictor in Part Two, respectively ${ }^{18}$ ) using readily-available computational tools, and 2) demonstrated the real-world applicability of this workflow by empirically assessing within-subject agreement between a free-list task and an item response scale on religious beliefs with a cross-culturally diverse sample. We failed to find evidence of a clear and consistent relationship between these two instruments, and discussed some possibilities as to what might explain this finding, at least in the particular domain of religious beliefs. At the very least, the presently reported results call for further research into the cognitive processes underlying item response scales and free-list tasks and pose an exciting challenge for field researchers, psychologists, and cognitive scientists to solve in future collaborative efforts. Another potential avenue for future research is to develop a principled and broadly implementable workflow for handling missing values in free-list tasks, accounting for uncertainty in an item's list position, as well as relating individual-level probability distributions of responses to a target population of interest (e.g., Deffner, Rohrer, \& McElreath, 2021).

Further, in terms of how to measure and model individual-level and cross-cultural variation in mental representations (e.g., beliefs, attitudes, values), we want to stress that the present work has ramifications that reach beyond the scholarly field in which the presented case study was conceived. For instance, there is arguably untapped potential in employing free-list tasks in measuring not only religious beliefs, but also beliefs and attitudes on science (e.g., Bendixen, 2020), morality (e.g., Purzycki, Pisor, et al., 2018; White \& Norenzayan, 2021), health (e.g., Quinlan, 2017), as well as political, ideological, and economic issues (e.g., Bendixen, 2019; White \& Norenzayan, 2021), disciplinary areas in which item response scales are extremely common but oftentimes not externally or cross-task verified. Here too, the free-list task could serve as a convenient measure of cross-task robustness or as a methodological tool to explore whether these two tasks indeed recruit distinct cognitive processes. As such, the original analysis reported herein is synergistic with the scope of the outlined tutorial; the reported null results emphasize a heightened need for attempts at cross-task verification of item response scales across disciplines. This in turn highlights the inevitability of engaging in detailed and rigorous ethnographic ${ }^{19}$ data collection and, thereby, motivates the dissemination of principled and implementable guidelines on how to model such data, including free-list outcomes, as alternative or complementary instruments to item response scales. 
More generally, zero-one bounded and inflated data are commonplace in many applied contexts. For instance, social scientists employ visual slider scales, "feeling thermometers", and other (semi-)continuous indices whereby participants rate their sentiments with regards to some item, claim, or public person, and medical scientists use "pain scales" to gauge patients' subjective levels of discomfort and pain. Moreover, proportions and rates are ubiquitous across the social and life sciences (e.g., Douma \& Weedon, 2019; Kubinec, 2022; P. Liu et al., 2020; Queiroz \& Lemonte, 2021; Santos \& Bolfarine, 2015). These and similar data would often have properties well-captured by the zero-one inflated beta distribution or the ordered beta distribution. We encourage researchers to carefully consider the processes that might have generated their data and, on this basis, fit several models of varying complexity and functional form following an iterative (Bayesian) workflow of model fitting, diagnosing, checking, comparison, tuning, and eventual inference (Gelman et al., 2020). Such a workflow does not have as its end goal the selection of a one "best" model; rather, like peering at white light through a prism, the successes and failures of fitting many different models often lead to insights easily overlooked by simply fitting one or a few. 


\section{Author contributions}

T.B. and B.G.P. conceptualized the manuscript. B.G.P. managed data collection for the greater project from which we analyzed a subset. T.B. wrote the manuscript and conducted all analyses with critical inputs from B.G.P.

\section{Acknowledgements}

We thank Aaron D. Lightner, Matti Vuorre, and two anonymous reviewers for comments on earlier drafts. We thank Aarhus University Research Foundation for financial support.

\section{Data Availability}

Data and code to reproduce this manuscript and the supplementary sections are available at: https://github.com/tbendixen/freelist-tutorial.

\section{References}

Allaire, J., Xie, Y., McPherson, J., Luraschi, J., Ushey, K., Atkins, A., .. Iannone, R. (2021). Rmarkdown: Dynamic documents for $r$. Retrieved from https://github.com/rst udio/rmarkdown

Apicella, C. L. (2018). High levels of rule-bending in a minimally religious and largely egalitarian forager population. Religion, Brain \& Behavior, 8(2), 133-148.

Atkinson, Q. D. (2018). Religion and expanding the cooperative sphere in kastom and christian villages on Tanna, Vanuatu. Religion, Brain \& Behavior, 8(2), 149-167.

Atkinson, Q. D., \& Bourrat, P. (2011). Beliefs about God, the afterlife and morality support the role of supernatural policing in human cooperation. Evolution and Human Behavior, 32(1), 41-49.

Atran, S., Medin, D., Ross, N., Lynch, E., Vapnarsky, V., Ek', E. U., ... Baran, M. (2002). Folkecology, Cultural Epidemiology, and the Spirit of the Commons: A Garden Experiment in the Maya Lowlands, 19912001. Current Anthropology, 43(3), 421-450. https://doi.org/10.1086/339528

Barrett, H. C. (2020). Deciding what to observe: Thoughts for a post-WEIRD generation. Evolution and Human Behavior, 41(5), 445-453. https://doi.org/10.1016/j.evolhumbeh av.2020.05.006

Bendixen, T. (2019). Sense or non-sense? A critical discussion of a recent evolutionarycognitive approach to "folk-economic beliefs." Evolution, Mind and Behaviour, 17(1), 29-47. https://doi.org/10.1556/2050.2019.00011

Bendixen, T. (2020). How cultural evolution can inform the science of science communicationand vice versa. Humanities and Social Sciences Communications, 7(1), 135. https: //doi.org/10.1057/s41599-020-00634-4

Bendixen, T., Apicella, C., Atkinson, Q., Cohen, E., Henrich, J., McNamara, R. A., ... Purzycki, B. G. (forthcoming). Appealing to the minds of gods: Religious beliefs and appeals correspond to features of local social ecologies. Religion, Brain $\&$ Behavior. Forthcoming. https://doi.org/10.31234/osf.io/tjn3e

Bendixen, T., Lightner, A. D., \& Purzycki, B. G. (forthcoming). The cultural evolution of religion and cooperation. In R. Kendal, J. Tehrani, \& J. Kendal (Eds.), Oxford handbook of cultural evolution. Oxford University Press. https://doi.org/10.31234/osf.io/fhscv 
Bendixen, T., \& Purzycki, B. G. (forthcoming). Accounting for cross-cultural variation in the minds of gods. In B. G. Purzycki \& T. Bendixen (Eds.), The minds of gods: New horizons in the naturalistic study of religion. Bloomsbury Publishing.

Bernard, H. R. (2018). Research methods in cultural anthropology. In Qualitative and (6th ed.).

Botero, C. A., Gardner, B., Kirby, K. R., Bulbulia, J., Gavin, M. C., \& Gray, R. D. (2014). The ecology of religious beliefs. Proceedings of the National Academy of Sciences, 111(47), 16784-16789. https://doi.org/10.1073/pnas.1408701111

Brewer, D. D., Garrett, S. B., \& Rinaldi, G. (2002). Free-listed items are effective cues for eliciting additional items in semantic domains. Applied Cognitive Psychology, 16 (3), 343-358. https://doi.org/https://doi.org/10.1002/acp.797

Broesch, T., Crittenden, A., Beheim, B., Blackwell, A., Bunce, J., Colleran, H., ... Mulder, M. (2020). Navigating cross-cultural research: Methodological and ethical considerations. Proceedings. Biological Sciences, 287, 20201245. https://doi.org/10.1098/rspb.2020.1245

Bunce, J. A., \& McElreath, R. (2017). Interethnic Interaction, Strategic Bargaining Power, and the Dynamics of Cultural Norms: A Field Study in an Amazonian Population. Human Nature, 28(4), 434-456. https://doi.org/10.1007/s12110-017-9297-8

Bürkner, P.-C. (2017). Brms: An R Package for Bayesian Multilevel Models Using Stan. Journal of Statistical Software, 80(1), 1-28. https://doi.org/10.18637/jss.v080.i01

Bürkner, P.-C. (2018). Advanced Bayesian Multilevel Modeling with the R Package brms. The R Journal, 10(1), 395-411. https://doi.org/10.32614/RJ-2018-017

Bürkner, P.-C. (2021). Bayesian item response modeling in $\mathrm{R}$ with brms and Stan. Journal of Statistical Software, 100(5), 1-54. https://doi.org/10.18637/jss.v100.i05

Bürkner, P.-C., \& Charpentier, E. (2020). Modelling monotonic effects of ordinal predictors in Bayesian regression models. British Journal of Mathematical and Statistical Psychology, 73 (3), 420-451. https://doi.org/https://doi.org/10.1111/bmsp.12195

Bürkner, P.-C., \& Vuorre, M. (2019). Ordinal regression models in psychology: A tutorial. Advances in Methods and Practices in Psychological Science, 2(1), 77-101. https: //doi.org/10.1177/2515245918823199

Carpenter, B., Gelman, A., Hoffman, M., Lee, D., Goodrich, B., Betancourt, M., .. Riddell, A. (2017). Stan: A probabilistic programming language. Journal of Statistical Software, $76(1)$.

Chaves, L. da S., Nascimento, A. L. B. do, Albuquerque, U. P., Chaves, L. da S., Nascimento, A. L. B. do, \& Albuquerque, U. P. (2019). What matters in free listing? A probabilistic interpretation of the salience index. Acta Botanica Brasilica, 33(2), 360-369. https: //doi.org/10.1590/0102-33062018abb0330

Cohen, E., Baimel, A., \& Purzycki, B. G. (2018). Religiosity and resource allocation in Marajó, Brazil. Religion, Brain Behavior, 8(2), 168-184. https://doi.org/10.1080/2153 599X.2016.1267029

Cribari-Neto, F., \& Santos, J. (2019). Inflated Kumaraswamy distributions. Anais Da Academia Brasileira de Ciências, 91. https://doi.org/10.1590/0001-3765201920180955

Curry, O., Whitehouse, H., \& Mullins, D. (2019). Is it good to cooperate? Testing the theory of morality-as-cooperation in 60 societies. Current Anthropology, 60(1).

D'Andrade, R. G. (1987). A folk model of the mind. In D. Holland \& N. Quinn (Eds.), Cultural models in language and thought (pp. 112-148). Cambridge University Press. 
https://doi.org/10.1017/CBO9780511607660.006

D'Andrade, R. G. (1995). The Development of Cognitive Anthropology. New York: Cambridge University Press.

Deffner, D., Rohrer, J. M., \& McElreath, R. (2021). A causal framework for cross-cultural generalizability. PsyArXiv. https://doi.org/10.31234/osf.io/fqukp

Douma, J. C., \& Weedon, J. T. (2019). Analysing continuous proportions in ecology and evolution: A practical introduction to beta and Dirichlet regression. Methods in Ecology and Evolution, 10(9), 1412-1430. https://doi.org/10.1111/2041-210X.13234

Dressler, W. W., Borges, C. D., Balieiro, M. C., \& Santos, J. E. dos. (2005). Measuring Cultural Consonance: Examples with Special Reference to Measurement Theory in Anthropology. Field Methods, 17(4), 331-355. https://doi.org/10.1177/1525822X052798 99

Erler, N. S., Rizopoulos, D., Rosmalen, J. van, Jaddoe, V. W. V., Franco, O. H., \& Lesaffre, E. M. E. H. (2016). Dealing with missing covariates in epidemiologic studies: A comparison between multiple imputation and a full Bayesian approach. Statistics in Medicine, 35(17), 2955-2974. https://doi.org/10.1002/sim.6944

Fremout, T., Gutiérrez-Miranda, C. E., Briers, S., Marcelo-Peña, J. L., Cueva-Ortiz, E., Linares-Palomino, R., ... Thomas, E. (2021). The value of local ecological knowledge to guide tree species selection in tropical dry forest restoration. Restoration Ecology, 29(4), e13347. https://doi.org/https://doi.org/10.1111/rec.13347

Furlow, C. A. (2003). Comparing indicators of knowledge within and between cultural domains. Field Methods, 15(1), 51-62.

Gabry, J., \& Mahr, T. (2021). Bayesplot: Plotting for bayesian models. Retrieved from https://mc-stan.org/bayesplot/

Gabry, J., Simpson, D., Vehtari, A., Betancourt, M., \& Gelman, A. (2019). Visualization in Bayesian workflow. Journal of the Royal Statistical Society: Series A (Statistics in Society), 182(2), 389-402. https://doi.org/10.1111/rssa.12378

Ge, E., Chen, Y., Wu, J., \& Mace, R. (2019). Large-scale cooperation driven by reputation, not fear of divine punishment. Royal Society Open Science, 6(8), 190991. https: //doi.org/10.1098/rsos.190991

Gelman, A., Carlin, J. B., Stern, D., Hal S, \& Rubin, D. B. (2013). Bayesian data analysis (3rd ed.). Chapman; Hall/CRC.

Gelman, A., Simpson, D., \& Betancourt, M. (2017). The prior can often only be understood in the context of the likelihood. Entropy, 19(10). https://doi.org/10.3390/e19100555

Gelman, A., Vehtari, A., Simpson, D., Margossian, C. C., Carpenter, B., Yao, Y., ... Modrák, M. (2020). Bayesian Workflow. arXiv:2011.01808 [Stat]. Retrieved from http://arxiv.org/abs/2011.01808

Graham, J., Haidt, J., Koleva, S., Motyl, M., Iyer, R., Wojcik, S. P., \& Ditto, P. H. (2013). Moral Foundations Theory: The Pragmatic Validity of Moral Pluralism. Advances in Experimental Social Psychology, 47, 55-130.

Haaf, J. M., Merkle, E. C., \& Rouder, J. N. (2020). Do items order? The psychology in IRT models. Journal of Mathematical Psychology, 98, 102398. https://doi.org/10.1016/j.jmp. 2020.102398

Handwerker, W. P. (2001). Quick ethnography. Lanham: Altamira Press.

Henley, N. M. (1969). A psychological study of the semantics of animal terms. Journal of 
Verbal Learning and Verbal Behavior, 8(2), 176-184. https://doi.org/https://doi.org/10 .1016/S0022-5371(69)80058-7

Jamieson-Lane, A., \& Purzycki, B. G. (2016). AnthroTools: Some custom tools for anthropology.

Johnson, A. A., Ott, M. Q., \& Dogucu, M. (2022). Bayes Rules! An Introduction to Applied Bayesian Modeling. Chapman; Hall/CRC Press.

Johnson, D. D. P. (2016). God is watching you: How the fear of god makes us human. Oxford University Press, USA.

Kay, M. (2022). tidybayes: Tidy data and geoms for Bayesian models. https://doi.org/10.5 $281 /$ zenodo. 1308151

Krosnick, J. A., \& Presser, S. (2009). Question and Questionnaire Design. In J. D. Wright \& P. V. Marsden (Eds.), Handbook of Survey Research (Second). San Diego, CA: Elsevier. Kruschke, J. K. (2014). Doing bayesian data analysis: A tutorial with r, JAGS, and stan.

Kruschke, J. K., \& Liddell, T. M. (2018). Bayesian data analysis for newcomers. Psychonomic Bulletin \& Review, 25(1), 155-177. https://doi.org/10.3758/s13423-017-1272-1

Kubinec, R. (2022). Ordered beta regression: A parsimonious, well-fitting model for continuous data with lower and upper bounds. Political Analysis. https://doi.org/10.101 $7 /$ pan.2022.20

Kundtová Klocová, E., Lang, M., Maňo, P., Kundt, R., \& Xygalatas, D. (2022). Cigarettes for the dead: Effects of sorcery beliefs on parochial prosociality in Mauritius. Religion, Brain \& Behavior, 12(1-2), 116-131. https://doi.org/10.1080/2153599X.2021.2006286

Kurz, A. S. (2021a). Doing Bayesian data analysis in brms and the tidyverse (version 0.4.0). Retrieved from https://bookdown.org/content/3686/

Kurz, A. S. (2021b). Statistical rethinking with brms, ggplot2, and the tidyverse: Second edition (version 0.2.0). Retrieved from https://bookdown.org/content/4857/

Lang, M., Purzycki, B. G., Apicella, C. L., Atkinson, Q. D., Bolyanatz, A., Cohen, E., ... others. (2019). Moralizing gods, impartiality and religious parochialism across 15 societies. Proceedings of the Royal Society B, 286(1898), 20190202.

Levine, J., Muthukrishna, M., Chan, K., \& Satterfield, T. (2015). Theories of the deep: Combining salience and network analyses to produce mental model visualizations of a coastal British Columbia food web. Ecology and Society, 20(4). https://doi.org/10.5751/ ES-08094-200442

Lewandowski, D., Kurowicka, D., \& Joe, H. (2009). Generating random correlation matrices based on vines and extended onion method. Journal of Multivariate Analysis, 100(9), $1989-2001$.

Liddell, T. M., \& Kruschke, J. K. (2018). Analyzing ordinal data with metric models: What could possibly go wrong? Journal of Experimental Social Psychology, 79, 328-348. https://doi.org/10.1016/j.jesp.2018.08.009

Liu, F., \& Eugenio, E. C. (2018). A review and comparison of bayesian and likelihood-based inferences in beta regression and zero-or-one-inflated beta regression. Statistical Methods in Medical Research, 27(4), 1024-1044. https://doi.org/10.1177/0962280216650699

Liu, F., \& Kong, Y. (2015). Zoib: An R Package for Bayesian Inference for Beta Regression and Zero/One Inflated Beta Regression. The $R$ Journal, 7(2), 34. https://doi.org/10.3 2614/RJ-2015-019

Liu, P., Yuen, K. C., Wu, L.-C., Tian, G.-L., \& Li, T. (2020). Zero-one-in ated simplex 
regression models for the analysis of continuous proportion data. Statistics and Its Interface, 13(2), 193-208. https://doi.org/10.4310/SII.2020.v13.n2.a5

McElreath, R. (2020). Statistical Rethinking: A Bayesian course with examples in $R$ and Stan (Second). CRC Press.

McNamara, R. A., \& Henrich, J. (2018). Jesus vs. The ancestors: How specific religious beliefs shape prosociality on Yasawa Island, Fiji. Religion, Brain \& Behavior, 8(2), 185-204.

McNamara, R. A., Senanayake, R., Willard, A. K., \& Henrich, J. (2021). God's mind on morality. Evolutionary Human Sciences, 3, E6. https://doi.org/10.1017/ehs.2021.1

McNamara, R. A., \& Wertz, A. E. (2021). Early Plant Learning in Fiji. Human Nature, 32(1), 115-149. https://doi.org/10.1007/s12110-021-09389-6

Menezes, A. F. B., Mazucheli, J., \& Bourguignon, M. (2021). A parametric quantile regression approach for modelling zero-or-one inflated double bounded data. Biometrical Journal, 63(4), 841-858. https://doi.org/10.1002/bimj.202000126

Mikkola, P., Martin, O. A., Chandramouli, S., Hartmann, M., Pla, O. A., Thomas, O., ... Klami, A. (2021). Prior knowledge elicitation: The past, present, and future. Retrieved from https://arxiv.org/abs/2112.01380

Norenzayan, A., Shariff, A. F., Gervais, W. M., Willard, A. K., McNamara, R. A., Slingerland, E., \& Henrich, J. (2016). The cultural evolution of prosocial religions. Behavioral and Brain Sciences, 39.

Ospina, R., \& Ferrari, S. L. P. (2012). A general class of zero-or-one inflated beta regression models. Computational Statistics \& Data Analysis, 56(6), 1609-1623. https://doi.org/ 10.1016/j.csda.2011.10.005

Panter, A. T., Tanaka, J. S., \& Wellens, T. R. (1992). The Psychometrics of Order Effects. In N. Schwarz \& S. Sudman (Eds.), Context Effects in Social and Psychological Research (pp. 249-264). New York, NY: Springer. https://doi.org/10.1007/978-1-4612-2848-6_17

Purzycki, B. G. (2011). Tyvan cher eezi and the socioecological constraints of supernatural agents' minds. Religion, Brain \&5 Behavior, 1(1), 31-45.

Purzycki, B. G. (2013). Toward a cognitive ecology of religious concepts: An example from the tyva republic. Journal for the Cognitive Science of Religion, 1(1), 99-120.

Purzycki, B. G. (forthcoming). Ethnographic free-list data: Management and analysis with examples in $R$. London: SAGE.

Purzycki, B. G., Apicella, C., Atkinson, Q. D., Cohen, E., McNamara, R. A., Willard, A. K., ... Henrich, J. (2016a). Cross-cultural dataset for the evolution of religion and morality project. Scientific Data, 3, 160099. https://doi.org/10.1038/sdata.2016.99

Purzycki, B. G., Apicella, C., Atkinson, Q. D., Cohen, E., McNamara, R. A., Willard, A. K., ... Henrich, J. (2016b). Moralistic gods, supernatural punishment and the expansion of human sociality. Nature, 530(7590), 327-330. https://doi.org/10.1038/nature16980

Purzycki, B. G., \& Bendixen, T. (2020). Examining Values, Virtues, and Tradition in the Tyva Republic with Free-List and Demographic Data. The New Research in Tuva, 4, 6-18.

Purzycki, B. G., Henrich, J., Apicella, C., Atkinson, Q. D., Baimel, A., Cohen, E., ... Norenzayan, A. (2018). The evolution of religion and morality: A synthesis of ethnographic and experimental evidence from eight societies. Religion, Brain $\&$ Behavior, $8(2), 101-132$. 
Purzycki, B. G., \& Holland, E. C. (2019). Buddha as a God: An Empirical Assessment. Method $\&$ Theory in the Study of Religion, 31(4-5), 347-375. https://doi.org/10.1163/15 $700682-12341453$

Purzycki, B. G., \& Jamieson-Lane, A. (2016). AnthroTools: An R package for cross-cultural ethnographic data analysis. Cross-Cultural Research, 51(1), 51-74.

Purzycki, B. G., \& Kulundary, V. (2018). Buddhism, identity, and class: Fairness and favoritism in the tyva republic. Religion, Brain \& Behavior, 8(2), 205-226.

Purzycki, B. G., \& McNamara, R. A. (2016). An ecological theory of gods' minds. In H. De Cruz \& R. Nichols (Eds.), Cognitive Science of Religion and Its Philosophical Implications (pp. 143-167). New York: Continuum.

Purzycki, B. G., Pisor, A., Apicella, C., Atkinson, Q. D., Cohen, E., Henrich, J., ... Xygalatas, D. (2018). The cognitive and cultural foundations of moral behavior. Evolution and Human Behavior, 39(5), 490-501.

Purzycki, B. G., Stagnaro, M. N., \& Sasaki, J. (2020). Breaches of Trust Change the Content and Structure of Religious Appeals. Journal for the Study of Religion, Nature and Culture, 14(1), 71-94. https://doi.org/10.1558/jsrnc.38786

Purzycki, B. G., Willard, A. K., Klocová, E. K., Apicella, C., Atkinson, Q., Bolyanatz, A., ... Ross, C. T. (2022). The moralization bias of gods' minds: A cross-cultural test. Religion, Brain \& Behavior, 12(1-2), 38-60. https://doi.org/10.1080/2153599X.2021.2006291

Queiroz, F. F., \& Lemonte, A. J. (2021). A broad class of zero-or-one inflated regression models for rates and proportions. Canadian Journal of Statistics, 49(2), 566-590. https://doi.org/10.1002/cjs.11576

Quinlan, M. B. (2005). Considerations for collecting freelists in the field: Examples from ethobotany. Field Methods, 17(3), 219-234.

Quinlan, M. B. (2017). The freelisting method. Handbook of Research Methods in Health Social Sciences. Singapore: Springer, 1-16.

R Core Team. (2021). R: A language and environment for statistical computing. Vienna, Austria: R Foundation for Statistical Computing. Retrieved from https://www.Rproject.org/

Redhead, Dr., Daniel, McElreath, R., \& Ross, C. T. (2021). Reliable network inference from unreliable data: A tutorial on latent network modeling using STRAND. PsyArXiv. https://doi.org/10.31234/osf.io/mkp2y

Rodríguez-Sánchez, F., Jackson, C. P., \& Hutchins, S. D. (2022). Grateful: Facilitate citation of $r$ packages. Retrieved from https://github.com/Pakillo/grateful

Romney, A. K., \& d'Andrade, R. G. (1964). Cognitive aspects of english kin terms. American Anthropologist, 66(3), 146-170.

Romney, A. K., Weller, S. C., \& Batchelder, W. H. (1986). Culture as consensus: A theory of culture and informant accuracy. American Anthropologist, 88(2), 313-338.

Şahin, M. D. (2021). Effect of Item Order on Certain Psychometric Properties: A Demonstration on a Cyberloafing Scale. Frontiers in Psychology, 12, 154 . https: //doi.org/10.3389/fpsyg.2021.590545

Santos, B., \& Bolfarine, H. (2015). Bayesian analysis for zero-or-one inflated proportion data using quantile regression. Journal of Statistical Computation and Simulation, 85(17), 3579-3593. https://doi.org/10.1080/00949655.2014.986733

Schloss, J. P., \& Murray, M. J. (2011). Evolutionary accounts of belief in supernatural 
punishment: A critical review. Religion, Brain \& Behavior, 1(1), 46-99.

Simpson, A., Piazza, J., \& Rios, K. (2016). Belief in divine moral authority: Validation of a shortened scale with implications for social attitudes and moral cognition. Personality and Individual Differences, 94, 256-265. https://doi.org/10.1016/j.paid.2016.01.032

Singh, M., \& Henrich, J. (2020). Why do religious leaders observe costly prohibitions? Examining taboos on Mentawai shamans. Evolutionary Human Sciences, 2. https: //doi.org/10.1017/ehs.2020.32

Singh, M., Kaptchuk, T. J., \& Henrich, J. (2021). Small gods, rituals, and cooperation: The Mentawai water spirit Sikameinan. Evolution and Human Behavior, 42(1), 61-72.

Smith, J. J. (1993). Using ANTHROPAC 3.5 and a Spreadsheet to Compute a Free-List Salience Index. CAM, 5(3), 1-3. https://doi.org/10.1177/1525822X9300500301

Smith, J. J., \& Borgatti, S. P. (1997). Salience Counts-And So Does Accuracy: Correcting and Updating a Measure for Free-List-Item Salience. Journal of Linguistic Anthropology, 7(2), 208-209. https://doi.org/10.1525/jlin.1997.7.2.208

Smith, J. J., Furbee, L., Maynard, K., Quick, S., \& Ross, L. (1995). Salience Counts: A Domain Analysis of English Color Terms. Journal of Linguistic Anthropology, 5(2), 203-216. https://doi.org/10.1525/jlin.1995.5.2.203

Stan Development Team. (2021). RStan: The $R$ interface to Stan. Retrieved from https://mc-stan.org/

Swearingen, C. J., Castro, M. M., \& Bursac, Z. (2012). Inflated beta regression: Zero, one, and everything in between. SAS Global Forum, 11. Citeseer.

Thompson, E. C., \& Juan, Z. (2006). Comparative cultural salience: Measures using free-list data. Field Methods, 18(4), 398-412. https://doi.org/10.1177/1525822X06293128

Turpin, H. D., \& Stanford, M. (2020). Cognitively informed ethnography: Using mixed methods to capture the complexity of religious phenomena in two ecologically valid settings. Journal for the Cognitive Science of Religion, 0(0). https://doi.org/10.1558/jc sr.38498

Ushey, K. (2022). Renv: Project environments. Retrieved from https://CRAN.R-project.or $\mathrm{g} /$ package $=$ renv

Vehtari, A., Gabry, J., Magnusson, M., Yao, Y., Bürkner, P.-C., Paananen, T., \& Gelman, A. (2020). Loo: Efficient leave-one-out cross-validation and WAIC for bayesian models. Retrieved from https://mc-stan.org/loo/

Vehtari, A., Gelman, A., \& Gabry, J. (2017). Practical Bayesian model evaluation using leave-one-out cross-validation and WAIC. Statistics and Computing, 27(5), 1413-1432. https://doi.org/10.1007/s11222-016-9696-4

Vuorre, M. (2019). How to analyze visual analog (slider) scale data? Retrieved from https://vuorre.netlify.app/post/2019/02/18/analyze-analog-scale-ratings-with-zeroone-inflated-beta-models/

Watts, J., Greenhill, S. J., Atkinson, Q. D., Currie, T. E., Bulbulia, J., \& Gray, R. D. (2015). Broad supernatural punishment but not moralizing high gods precede the evolution of political complexity in Austronesia. Proceedings of the Royal Society of London B: Biological Sciences, 282(1804), 20142556. https://doi.org/10.1098/rspb.2014.2556

White, C. J. M., \& Norenzayan, A. (2021). Karma and God: Convergent and divergent mental representations of supernatural norm enforcement. Psychology of Religion and Spirituality. https://doi.org/10.1037/rel0000436 
Wickham, H. (2016). ggplot2: Elegant graphics for data analysis. Springer-Verlag New York. Retrieved from https://ggplot2.tidyverse.org

Wickham, H., Averick, M., Bryan, J., Chang, W., McGowan, L. D., François, R., ... Yutani, H. (2019). Welcome to the tidyverse. Journal of Open Source Software, 4(43), 1686. https://doi.org/10.21105/joss.01686

Willard, A. K. (2018). Religion and prosocial behavior among the indo-fijians. Religion, Brain \& Behavior, 8(2), 227-242. https://doi.org/10.1080/2153599X.2016.1267032

Willard, A. K., Baimel, A., Turpin, H., Jong, J., \& Whitehouse, H. (2020). Rewarding the good and punishing the bad: The role of karma and afterlife beliefs in shaping moral norms. Evolution and Human Behavior, 41(5), 385-396. https://doi.org/10.1016/j.evol humbehav.2020.07.001

Wood, S. N. (2017). Generalized Additive Models: An Introduction with $R$ (Second). Chapman; Hall/CRC.

Xie, Y., Allaire, J. J., \& Grolemund, G. (2018). $R$ markdown: The definitive guide. Boca Raton, Florida: Chapman; Hall/CRC. Retrieved from https://bookdown.org/yihui/rma rkdown

Xygalatas, D., Kotherová, S., Maňo, P., Kundt, R., Cigán, J., Klocová, E. K., \& Lang, M. (2018). Big gods in small places: The random allocation game in mauritius. Religion, Brain \& Behavior, 8(2), 243-261. https://doi.org/10.1080/2153599X.2016.1267033 